\title{
dftatom: A robust and general Schrödinger and Dirac solver for atomic structure calculations
}

\author{
Ondřej Čertík ${ }^{a, b, c}$, John E. Pask ${ }^{d}$, Jiří Vackářa \\ ${ }^{a}$ Institute of Physics, Academy of Sciences of the Czech Republic, Na Slovance 2, \\ 182 21 Praha 8, Czech Republic \\ ${ }^{\mathrm{b}}$ University of Nevada, Reno - 1664 N. Virginia St., Reno, NV 89557-0208, USA \\ ${ }^{\mathrm{c}}$ Faculty of Mathematics and Physics, Charles University, Ke Karlovu 3, 12116 \\ Praha 2, Czech Republic \\ ${ }^{\mathrm{d}}$ Lawrence Livermore National Laboratory, 7000 East Avenue, Livermore, CA \\ 94550, USA
}

\begin{abstract}
A robust and general solver for the radial Schrödinger, Dirac, and Kohn-Sham equations is presented. The formulation admits general potentials and meshes: uniform, exponential, or other defined by nodal distribution and derivative functions. For a given mesh type, convergence can be controlled systematically by increasing the number of grid points. Radial integrations are carried out using a combination of asymptotic forms, Runge-Kutta, and implicit Adams methods. Eigenfunctions are determined by a combination of bisection and perturbation methods for robustness and speed. An outward Poisson integration is employed to increase accuracy in the core region, allowing absolute accuracies of $10^{-8}$ Hartree to be attained for total energies of heavy atoms such as uranium. Detailed convergence studies are presented and computational parameters are provided to achieve accuracies commonly required in practice. Comparisons to analytic and current-benchmark density-functional results for atomic number $Z=1-92$ are presented, verifying and providing a refinement to current benchmarks. An efficient, modular Fortran 95 implementation, dftatom, is provided as open source, including examples, tests, and wrappers for interface to other languages; wherein particular emphasis is placed on the independence (no global variables), reusability, and generality of the individual routines.
\end{abstract}

Key words: atomic structure, electronic structure, Schrödinger equation, Dirac equation, Kohn-Sham equations, density functional theory, shooting method, Fortran 95, Python, C

PACS: 


\section{PROGRAM SUMMARY}

Manuscript Title: dftatom: A robust and general Schrödinger and Dirac solver for atomic structure calulations

Authors: Ondřej Čertík, John E. Pask, Jiří Vackář

Program Title: dftatom

Journal Reference:

Catalogue identifier:

Licensing provisions: MIT license

Programming language: Fortran 95 with interfaces to Python and $\mathrm{C}$

Computer: Any computer with Fortran 95 compiler

Operating system: Any OS with Fortran 95 compiler

RAM: $500 \mathrm{MB}$

Number of processors used:

Keywords:

atomic structure, electronic structure, Schrödinger equation, Dirac equation, KohnSham equations, density functional theory, shooting method, Fortran 95, Python, $\mathrm{C}$

Classification: 2.1 Structure and Properties

Nature of problem:

Solution of the Schrödinger, Dirac, and Kohn-Sham equations of Density Functional Theory for isolated atoms.

Solution method:

Radial integrations are carried out using a combination of asymptotic forms, RungeKutta, and implicit Adams methods. Eigenfunctions are determined by a combination of bisection and perturbation methods. An outward Poisson integration is employed to increase accuracy in the core region. Self-consistent field equations are solved by adaptive linear mixing.

Restrictions: Spherical symmetry

Unusual features:

Radial integrators work for general potentials and meshes. No restriction to Coulombic or self-consistent potentials; no restriction to uniform or exponential meshes. Outward Poisson integration. Fallback to bisection for robustness.

Additional comments:

Running time: For uranium, non-relativistic density functional calculation execution time is around $0.6 \mathrm{~s}$ for $10^{-6}$ a.u. accuracy in total energy on Intel Core i7 $1.46 \mathrm{GHz}$ processor.

Email addresses: ondrej.certik@gmail.com (Ondřej Čertík), pask1@llnl.gov (John E. Pask), vackar@fzu.cz (Jiří Vackář). 


\section{Introduction}

Atomic structure calculations are a lynchpin of modern materials theory. They provide the basis for understanding the properties of individual atoms and play a central role in electronic structure calculations of larger, multi-atom systems such as molecules, nanostructures, and solids [1]. In the latter context, being a core computational kernel for larger-scale calculations, both accuracy and efficiency are of prime importance. Such atomic calculations, solving radial Schrödinger or Dirac equations, can be found at the heart of plasma physics calculations [2], all-electron electronic structure methods such as Korringa-Kohn-Rostoker (KKR) [3], linearized muffin tin orbital (LMTO) [4], and linearized augmented planewave (LAPW) [5], pseudo-atomic-orbital based methods [6], ab initio pseudopotential construction [1], and projector augmented wave (PAW) [7], relaxed-core PAW [8], and all-electron pseudopotential (AEPP) [9] methods, among others.

Due to their central importance in the full range of electronic structure calculations, from isolated atoms to solids, a number of atomic structure codes have been developed over the decades, see, e.g., [10-16]. However, most have been developed within, and remain closely tied to, the specific larger-scale method of which they are a part, and hence are difficult to separate and use for other purposes. Indeed, it was precisely our own need for a robust and general solver for use in a finite-element [17] based AEPP [9] method that motivated the present work. Beyond difficulty to extract, however, existing codes have widely differing capabilities, efficiencies, robustness, and availability. Table 1 lists features of several established codes. Some solve the equations of density functional theory (DFT) while others solve Hartree-Fock (HF). Some are relativistic, others not. Some can be converged to high precision while others can be difficult to converge beyond the accuracies required by the larger codes of which they are a part. Some allow any mesh while others implement only exponential meshes. Some allow any potential while others implement only singular all-electron potentials. Some implement perturbation corrections for speed, others only bisection. Some are open source, others not. Note that the table shows only shooting-type solvers [18], as we discuss in the present work. Other non-shooting type approaches, see, e.g., [19-26] and references therein, have been developed and implemented as well but have not yet found wide adoption in larger-scale electronic structure calculations due in part to robustness issues (e.g., spurious states) which can arise [26]. Note also that the table is intended only as a general guide: in the context of any given feature, "No"

should be understood to mean only that we did not find it straightforward to implement in the referenced code.

In the present work, we bring together ideas from a host of atomic structure codes developed over many decades, and add new ideas to increase ac- 


\begin{tabular}{lcccccccc} 
Code & dftatom & Desclaux & atompp & grasp2k & MCHF & atompaw & PEtot & Elk \\
\hline Reference & This work & {$[10]$} & {$[11]$} & {$[12]$} & {$[13]$} & {$[14]$} & {$[15]$} & {$[16]$} \\
Open Source & MIT & No & No & No & No & GPL & BSD & GPL \\
Schrödinger & Yes & No & Yes & No & Yes & Yes & Yes & No \\
Dirac & Yes & Yes & No & Yes & No & No & Yes & Yes \\
DFT & Yes & No & Yes & No & No & Yes & Yes & Yes \\
Any potential & Yes & No & Yes & No & No & No & No & Yes \\
Any mesh & Yes & No & No & No & No & No & No & Yes \\
Outward Poisson & Yes & No & No & No & No & No & No & No \\
Perturb. correct. & Yes & Yes & Yes & No & Yes & Yes & Yes & No \\
Bisection fallback & Yes & No & No & No & No & No & No & No \\
\hline
\end{tabular}

Table 1

Features of various atomic structure solvers.

curacy and robustness. We try to do this in such a way as to provide as general, flexible, and efficient a tool as possible, facilitating subsequent use in a range of applications areas. We develop and implement solvers for the radial Schrödinger, Dirac, and Kohn-Sham equations. The formulation allows general potentials and meshes: singular Coulomb-, finite pseudo-, or other potentials; uniform, exponential, or other meshes, as defined by nodal distributions and derivatives. For a given mesh type, convergence can be controlled systematically by increasing the number of grid points. Radial integrations are carried out using a combination of asymptotic forms, Runge-Kutta, and implicit Adams methods. Eigenfunctions are determined by a combination of bisection and perturbation methods for robustness and speed. Unlike other available codes [10-16], we employ an outward Poisson integration to increase accuracy in the core region, allowing absolute accuracies of $10^{-8}$ Hartree to be attained for total energies of heavy atoms such as uranium. We show detailed convergence studies and provide computational parameters to achieve accuracies commonly required in practice. We present comparisons to analytic and current-benchmark density-functional results $[27,28]$ for atomic number $Z=$ $1-92$, verifying and refining current benchmarks.

We provide an efficient, modular Fortran 95 implementation, dftatom, as open source, including examples, tests, and wrappers for interface to other languages. The code consists of several clearly arranged Fortran 95 modules, designed to be easy to apply for other purposes. The integration routines are independent of mesh, which can be specified as desired. Routines for common meshes are provided. Extensive tests are included to verify the correctness of results and serve as examples for a number of cases: Coulombic, self consistent DFT, and non-singular potentials; exponential and hyperbolic meshes; atomic numbers $Z=1-92$; user-specified occupation numbers; and others. The code is opensource, available under the terms of the MIT license, from [29]. C and Python wrappers are provided so that the code can be easily used from other languages as well as interactively from Python. 
The remainder of the paper is organized as follows. Section 2 describes the radial Schrödinger, Dirac, and Poisson equations, associated asymptotics, and DFT equations to be solved. Section 3 describes the numerical methods used to solve the radial equations and eigenproblem, and meshes employed. Section 4 shows Schrödinger and Dirac results for singular and nonsingular potentials, relativistic and nonrelativistic DFT calculations of uranium, convergence studies determining parameters for specified accuracies, and comparison to established benchmarks [27]. We conclude in Section 5.

\section{Equations}

\subsection{Radial Schrödinger equation}

The 3D one-electron Schrödinger equation is given by

$$
\left(-\frac{1}{2} \nabla^{2}+V(\mathbf{x})\right) \psi(\mathbf{x})=E \psi(\mathbf{x})
$$

in Hartree atomic units, as we use throughout. For a spherically symmetric potential

$$
V(\mathbf{x})=V(r)
$$

eigenstates of energy and angular momentum can be written in the form

$$
\psi_{n l m}(\mathbf{x})=R_{n l}(r) Y_{l m}\left(\frac{\mathbf{x}}{r}\right)
$$

where $n$ is the principal quantum number, $l$ is the orbital angular momentum quantum number, and $m$ is the magnetic quantum number; whereupon it follows that $R_{n l}(r)$ satisfies the radial Schrödinger equation

$$
\left(-\frac{1}{2} r^{2} R_{n l}^{\prime}(r)\right)^{\prime}+\left(r^{2} V+\frac{1}{2} l(l+1)\right) R_{n l}(r)=E r^{2} R_{n l}(r) .
$$

The functions $\psi_{n l m}(\mathbf{x})$ and $R_{n l}(r)$ are normalized as

$$
\begin{gathered}
\int\left|\psi_{n l m}(\mathbf{x})\right|^{2} \mathrm{~d}^{3} x=1 \\
\int_{0}^{\infty} R_{n l}^{2}(r) r^{2} \mathrm{~d} r=1
\end{gathered}
$$

The substitution $P_{n l}(r)=r R_{n l}(r)$ and $Q_{n l}(r)=P_{n l}^{\prime}(r)=R_{n l}(r)+r R_{n l}^{\prime}(r)$ can be used to write the second-order radial Schrödinger equation as a coupled set of first-order equations:

$$
\begin{aligned}
P_{n l}^{\prime}(r) & =Q_{n l}(r), \\
Q_{n l}^{\prime}(r) & =2\left(V(r)-E+\frac{l(l+1)}{2 r^{2}}\right) P_{n l}(r) .
\end{aligned}
$$


Such a first-order formulation facilitates solving both nonrelativistic Schrödinger and relativistic Dirac equations using the same techniques. The normalization of $P(r)$ is

$$
\int_{0}^{\infty} P_{n l}^{2}(r) \mathrm{d} r=1
$$

For a potential $V(r)$ which behaves like $-Z / r+V_{0}$ near the origin, as in the vicinity of the nucleus, the asymptotic behaviors of $P_{n l}$ and $Q_{n l}$ are [30]

$$
\begin{aligned}
P_{n l}(r) & =r^{l+1} \\
Q_{n l}(r) & =(l+1) r^{l} .
\end{aligned}
$$

For large $r$, assuming $V(r) \rightarrow 0$ as $r \rightarrow \infty$, the asymptotic is [30]:

$$
\begin{aligned}
P_{n l}(r) & =e^{-\lambda r}, \\
Q_{n l}(r) & =-\lambda P_{n l}(r),
\end{aligned}
$$

where

$$
\lambda=\sqrt{-2 E_{n l}}
$$

As solutions of a homogeneous system, $P_{n l}$ and $Q_{n l}$ are unique only up to an arbitrary multiplicative constant. These small- and large- $r$ asymptotics provide starting values and derivatives for inward and outward numerical integrations.

\subsection{Radial Dirac equation}

The one-electron radial Dirac equation can be written as

$$
\begin{aligned}
& P_{n \kappa}^{\prime}(r)=-\frac{\kappa}{r} P_{n \kappa}(r)+\left(\frac{E-V(r)}{c}+2 c\right) Q_{n \kappa}(r), \\
& Q_{n \kappa}^{\prime}(r)=-\left(\frac{E-V(r)}{c}\right) P_{n \kappa}(r)+\frac{\kappa}{r} Q_{n \kappa}(r),
\end{aligned}
$$

where $P_{n \kappa}(r)$ and $Q_{n \kappa}(r)$ are related to the usual large $g_{n \kappa}(r)$ and small $f_{n \kappa}(r)$ components of the Dirac equation by

$$
\begin{aligned}
P_{n \kappa}(r) & =r g_{n \kappa}(r), \\
Q_{n \kappa}(r) & =r f_{n \kappa}(r) .
\end{aligned}
$$

See for example [31,32] for a discussion and derivation. The solutions of the Dirac equation are labeled by quantum numbers $n$ and $\kappa$, where $n$ is the principal quantum number and $\kappa \neq 0$ is an integer. Alternatively, they may be labeled by the triplet $n, l, s$, where $l$ is the orbital angular momentum and $s= \pm 1$ distinguishes the $j=l \pm \frac{1}{2}$ states ( $j$ is the total angular momentum). 
$\kappa$ is then determined by

$$
\kappa=\left\{\begin{array}{cl}
-l-1 & \text { for } j=l+\frac{1}{2}, \text { i.e. } s=+1 \\
l & \text { for } j=l-\frac{1}{2}, \text { i.e. } s=-1
\end{array}\right.
$$

The energy $E$ does not contain the electron rest mass energy, so it can be compared directly to the corresponding nonrelativistic energy of the Schrödinger equation. The normalization of $P_{n \kappa}(r)$ and $Q_{n \kappa}(r)$ is

$$
\int_{0}^{\infty}\left(P_{n \kappa}^{2}(r)+Q_{n \kappa}^{2}(r)\right) \mathrm{d} r=1 .
$$

For potentials of the form $V(r)=-Z / r+Z_{1}+O(r)$, the asymptotic behavior at the origin for $Z \neq 0$ (Coulombic) is [33,32]

$$
\begin{aligned}
& P_{n \kappa}(r)=r^{\beta}, \\
& Q_{n \kappa}(r)=r^{\beta} \frac{c(\beta+\kappa)}{Z},
\end{aligned}
$$

where

$$
\beta=\sqrt{\kappa^{2}-\left(\frac{Z}{c}\right)^{2}} .
$$

For $Z=0$ (nonsingular) the asymptotic is [33], for $\kappa<0$

$$
\begin{aligned}
P_{n \kappa}(r) & =r^{l+1} \\
Q_{n \kappa}(r) & =r^{l+2} \frac{E+Z_{1}}{c(2 l+3)},
\end{aligned}
$$

and for $\kappa>0$

$$
\begin{aligned}
P_{n \kappa}(r) & =-r^{l+2} \frac{E+Z_{1}}{c(2 l+1)}, \\
Q_{n \kappa}(r) & =r^{l+1} .
\end{aligned}
$$

For large $r$, assuming $V(r) \rightarrow 0$ as $r \rightarrow \infty$, the asymptotic is [30]

$$
\begin{aligned}
P_{n \kappa}(r) & =e^{-\lambda r}, \\
Q_{n \kappa}(r) & =-\sqrt{-\frac{E}{E+2 c^{2}}} P_{n \kappa}(r),
\end{aligned}
$$

where

$$
\lambda=\sqrt{c^{2}-\frac{\left(E+c^{2}\right)^{2}}{c^{2}}}=\sqrt{-2 E-\frac{E^{2}}{c^{2}}} .
$$

As in the case of the Schrödinger equation, $P_{n \kappa}$ and $Q_{n \kappa}$ are unique only up to an arbitrary multiplicative constant, and the above asymptotics provide starting values and derivatives for inward and outward numerical integrations. 


\subsection{Poisson equation}

The 3D Poisson equation for the Hartree potential $V_{H}$ due to electronic density $n$ is given by

$$
\nabla^{2} V_{H}(\mathbf{x})=-4 \pi n(\mathbf{x})
$$

For a spherical density $n(\mathbf{x})=n(r)$, this becomes

$$
\frac{1}{r^{2}}\left(r^{2} V_{H}^{\prime}\right)^{\prime}=V_{H}^{\prime \prime}(r)+\frac{2}{r} V_{H}^{\prime}(r)=-4 \pi n(r),
$$

where $n(r)$ is the radial particle (number) density, normalized such that

$$
N=\int n(\mathbf{x}) \mathrm{d}^{3} x=\int_{0}^{\infty} 4 \pi n(r) r^{2} \mathrm{~d} r
$$

where $N$ is the electron number. Substituting (33) into (34) and integrating, we obtain

$$
\lim _{r \rightarrow \infty} r^{2} V_{H}^{\prime}(r)=-N
$$

from which it follows that the asymptotic behavior of $V_{H}^{\prime}(r)$ is

$$
V_{H}^{\prime}(r)=-\frac{N}{r^{2}}, \quad r \rightarrow \infty .
$$

Integrating (36) and requiring $V_{H} \rightarrow 0$ as $r \rightarrow \infty$ then gives the corresponding asymptotic behavior for $V_{H}(r)$ :

$$
V_{H}(r)=\frac{N}{r}, \quad r \rightarrow \infty
$$

For small $r$, the asymptotic behavior can be obtained by expanding $n(r)$ about $r=0: n(r)=\sum_{j=0}^{\infty} c_{j} r^{j}$. Substituting into Poisson equation (33) gives

$$
\left(r^{2} V_{H}^{\prime}\right)^{\prime}=-4 \pi \sum_{j=0}^{\infty} c_{j} r^{j+2}
$$

Integrating and requiring $V_{H}(0)$ finite then gives

$$
V_{H}^{\prime}(r)=-4 \pi \sum_{j=0}^{\infty} c_{j} \frac{r^{j+1}}{j+3},
$$

with linear leading term; so that we have

$$
V_{H}^{\prime}(r) \propto r, \quad r \rightarrow 0
$$

Integrating (39) then gives

$$
V_{H}(r)=-4 \pi \sum_{j=0}^{\infty} c_{j} \frac{r^{j+2}}{(j+2)(j+3)}+C,
$$


with leading constant term $C=V_{H}(0)$ determined by Coulomb's law:

$$
V_{H}(0)=4 \pi \int_{0}^{\infty} r n(r) \mathrm{d} r .
$$

Finally, from (40) we have that

$$
V_{H}^{\prime}(0)=0
$$

The above asymptotics provide starting values and derivatives for inward and outward numerical integrations.

\subsection{Kohn-Sham equations}

The Kohn-Sham equations consist of the radial Schrödinger or Dirac equations above with an effective potential $V(r)=V_{\text {in }}(r)$ given by (see, e.g., [1])

$$
V_{\text {in }}=V_{H}+V_{x c}+v
$$

where $V_{H}$ is the Hartree potential given by the solution of the radial Poisson equation (33), $V_{x c}$ is the exchange-correlation potential and $v=-\frac{Z}{r}$ is the nuclear potential.

The total energy is given by

$$
E[n]=T_{s}[n]+E_{H}[n]+E_{x c}[n]+V[n],
$$

the sum of kinetic energy

$$
T_{s}[n]=\sum_{n l} f_{n l} \epsilon_{n l}-4 \pi \int V_{\text {in }}(r) n(r) r^{2} \mathrm{~d} r,
$$

where $\epsilon_{n l}$ are the Kohn-Sham eigenvalues, Hartree energy

$$
E_{H}[n]=2 \pi \int V_{H}(r) n(r) r^{2} \mathrm{~d} r
$$

exchange-correlation energy

$$
E_{x c}[n]=4 \pi \int \epsilon_{x c}(r ; n) n(r) r^{2} \mathrm{~d} r
$$

where $\epsilon_{x c}(r ; n)$ is the exchange and correlation energy density, and Coulomb energy

$$
V[n]=4 \pi \int v(r) n(r) r^{2} \mathrm{~d} r=-4 \pi Z \int n(r) r \mathrm{~d} r
$$

with electronic density in the nonrelativistic case given by

$$
n(r)=\frac{1}{4 \pi} \sum_{n l} f_{n l} \frac{P_{n l}^{2}(r)}{r^{2}},
$$


where $P_{n l}$ is the radial wavefunction (Eq. (7)) and $f_{n l}$ the associated electronic occupation. In the relativistic case, the electronic density is given by

$$
n(r)=\frac{1}{4 \pi} \sum_{n l s} f_{n l s} \frac{P_{n l s}^{2}(r)+Q_{n l s}^{2}(r)}{r^{2}},
$$

where $P_{n l s}$ and $Q_{n l s}$ are the two components of the Dirac solution (Eqs. (15), (16)) and $f_{n l s}$ is the occupation. In both cases above, $n(r)$ is the electronic particle density [electrons/volume], everywhere positive, as distinct from the electronic charge density $\rho(r)$ [charge/volume]: $\rho(r)=-n(r)$ in atomic units.

These equations are solved self-consistently (see, e.g., [1]): an initial density $n_{\text {in }}$ and corresponding potential $V_{\text {in }}$ are constructed; the Schrödinger or Dirac equation is solved to determine wavefunctions $R_{n l}$ or spinor components $P$ and $Q$, respectively; from these a new density $n_{\text {out }}$ and potential $V_{\text {out }}$ are constructed; and from these, a new input density $n_{\text {in }}$ and potential $V_{\text {in }}$ are constructed. The process is continued until the difference of $V_{\text {in }}$ and $V_{\text {out }}$ and/or $n_{\text {in }}$ and $n_{\text {out }}$ is within a specified tolerance, at which point self-consistency is achieved. This fixed point iteration is known as the self-consistent field (SCF) iteration. We employ an adaptive linear mixing scheme, with optimized weights for each component of the potential to construct new input potentials for successive SCF iterations. In order to reduce the number of SCF iterations, we use a Thomas-Fermi (TF) approximation [34] for the initial density and potential:

where

$$
V(r)=-\frac{Z_{\mathrm{eff}}(r)}{r}
$$

$$
\begin{gathered}
Z_{\mathrm{eff}}(r)=Z\left(1+\alpha \sqrt{x}+\beta x e^{-\gamma \sqrt{x}}\right)^{2} e^{-2 \alpha \sqrt{x}}, \\
x=r\left(\frac{128 Z}{9 \pi^{2}}\right)^{\frac{1}{3}} \\
\alpha=0.7280642371 \\
\beta=-0.5430794693 \\
\gamma=0.3612163121 .
\end{gathered}
$$

The corresponding charge density is then

$$
\rho(r)=-\frac{1}{3 \pi^{2}}(-2 V(r))^{\frac{3}{2}} .
$$

For simplicity, we consider only local-density approximation (LDA) and relativistic local-density approximation (RLDA) exchange and correlation potentials here. More sophisticated functionals can be readily incorporated. Our dftatom implementation uses the same parameterization as in NIST benchmarks [27]:

$$
V_{x c}(r ; n)=\frac{\mathrm{d}}{\mathrm{d} n}\left(n \epsilon_{x c}^{L D}(n)\right),
$$


where the exchange and correlation energy density $\epsilon_{x c}^{L D}$ can be written as [1]

$$
\epsilon_{x c}^{L D}(n)=\epsilon_{x}^{L D}(n)+\epsilon_{c}^{L D}(n),
$$

with electron gas exchange term [1]

$$
\epsilon_{x}^{L D}(n)=-\frac{3}{4 \pi}\left(3 \pi^{2} n\right)^{\frac{1}{3}}
$$

and Vosko-Wilk-Nusair (VWN) [35] correlation term

$$
\begin{aligned}
\epsilon_{c}^{L D}(n) \approx & \frac{A}{2}\left\{\log \left(\frac{y^{2}}{Y(y)}\right)+\frac{2 b}{Q} \arctan \left(\frac{Q}{2 y+b}\right)\right. \\
& \left.-\frac{b y_{0}}{Y\left(y_{0}\right)}\left[\log \left(\frac{\left(y-y_{0}\right)^{2}}{Y(y)}\right)+\frac{2\left(b+2 y_{0}\right)}{Q} \arctan \left(\frac{Q}{2 y+b}\right)\right]\right\},
\end{aligned}
$$

in which $y=\sqrt{r_{s}}, Y(y)=y^{2}+b y+c, Q=\sqrt{4 c-b^{2}}, y_{0}=-0.10498$, $b=3.72744, c=12.9352, A=0.0621814$, and

$$
r_{s}=\left(\frac{3}{4 \pi n}\right)^{\frac{1}{3}}
$$

is the Wigner-Seitz radius, which gives the mean distance between electrons. In the relativistic (RLDA) case, a correction to the LDA exchange energy density and potential is given by MacDonald and Vosko [36]:

$$
\begin{gathered}
\epsilon_{x}^{R L D}(n)=\epsilon_{x}^{L D}(n) R, \\
R=1-\frac{3}{2}\left(\frac{\beta \mu-\log (\beta+\mu)}{\beta^{2}}\right)^{2}, \\
V_{x}^{R L D}(n)=V_{x}^{L D}(n) S, \\
S=\frac{3 \log (\beta+\mu)}{2 \beta \mu}-\frac{1}{2},
\end{gathered}
$$

where $\mu=\sqrt{1+\beta^{2}}$ and $\beta=\frac{\left(3 \pi^{2} n\right)^{\frac{1}{3}}}{c}=-\frac{4 \pi \epsilon_{x}^{L D}(n)}{3 c}$.

\section{Methods of solution}

In this section, we discuss the radial integration methods, meshes, and eigenfunction isolation methods employed in the Kohn-Sham solution. 


\subsection{Runge-Kutta and Adams methods}

To allow general, nonuniform meshes, all methods first transform equations on a general mesh $R(t), 1 \leq t \leq N+1$, to equations on a uniform mesh $t$ with step size $h=1$. If the solution on a general mesh is $P(r)$ and the transformed solution on the uniform mesh is $u(t)$ then

$$
\begin{aligned}
u(t) & =P(R(t)) \\
u^{\prime}(t) & =\frac{\mathrm{d} u}{\mathrm{~d} t}=\frac{\mathrm{d} P}{\mathrm{~d} R} R^{\prime}(t)
\end{aligned}
$$

The methods below require the values $u(t)$ and derivatives $u^{\prime}(t)$ on the uniform mesh, which we express in terms of the values $P(r)$ and derivatives $P^{\prime}(r)$ on the general mesh, and derivative $R^{\prime}(t)$ of the function defining the general mesh.

The Runge-Kutta family of methods for the numerical integration of ordinary differential equations require the values of dependent and independent variables in the interior of the element (step). For example, the 4th-order Runge-Kutta (RK4) step for an equation of the form $y^{\prime}=f(x, y)$ is:

$$
\begin{aligned}
y_{i+1} & =y_{i}+\frac{1}{6}\left(k_{1}+2 k_{2}+2 k_{3}+k_{4}\right), \\
k_{1} & =f\left(x_{i}, y_{i}\right) \\
k_{2} & =f\left(x_{i+\frac{1}{2}}, y_{i}+\frac{1}{2} k_{1}\right), \\
k_{3} & =f\left(x_{i+\frac{1}{2}}, y_{i}+\frac{1}{2} k_{2}\right), \\
k_{4} & =f\left(x_{i+1}, y_{i}+k_{3}\right) .
\end{aligned}
$$

Implicit Adams methods, on the other hand, use an extrapolation formula to advance the solution to the next grid point and then an interpolation formula to correct the value (predictor-corrector) using the grid points only. The 4thorder Adams outward extrapolation is given by

$$
y_{i+1}=y_{i}+\frac{1}{24}\left(55 y_{i}^{\prime}-59 y_{i-1}^{\prime}+37 y_{i-2}^{\prime}-9 y_{i-3}^{\prime}\right)
$$

and interpolation by

$$
y_{i+1}=y_{i}+\frac{1}{24}\left(9 y_{i+1}^{\prime}+19 y_{i}^{\prime}-5 y_{i-1}^{\prime}+y_{i-2}^{\prime}\right) .
$$

Since the Schrödinger and Dirac equations are linear (for given effective potential in each SCF iteration), the interpolation can be determined analytically, thus eliminating predictor-corrector iterations, speeding up the calculation 
considerably. Both Schrödinger and Dirac equations can be written as

$$
\left(\begin{array}{l}
P^{\prime} \\
Q^{\prime}
\end{array}\right)=C\left(\begin{array}{l}
P \\
Q
\end{array}\right)
$$

where for the Schrödinger equation, the matrix $C$ is given by

$$
C(i)=\left(\begin{array}{cc}
0 & 1 \\
\frac{l(l+1)}{R^{2}(i)}+2(V(i)-E) & 0
\end{array}\right)
$$

and for the Dirac equation,

$$
C(i)=\left(\begin{array}{cc}
-\frac{\kappa}{R(i)} & \frac{E-V(i)}{c}+2 c \\
-\frac{E-V(i)}{c} & \frac{\kappa}{R(i)}
\end{array}\right) .
$$

Then for outward integration, analytic interpolation gives:

$$
\begin{aligned}
\lambda & =\frac{9}{24}, \\
\Delta & =1+\lambda^{2} R^{\prime 2}(i+1) \operatorname{det} C(i+1), \\
M & =\frac{1}{\Delta}\left(\left(\begin{array}{ll}
1 & 0 \\
0 & 1
\end{array}\right)+\lambda R^{\prime}(i+1)\left(\begin{array}{cc}
-C_{22}(i+1) & C_{12}(i+1) \\
C_{21}(i+1) & -C_{11}(i+1)
\end{array}\right)\right), \\
\left(\begin{array}{l}
u_{1}(i+1) \\
u_{2}(i+1)
\end{array}\right) & =M\left(\begin{array}{l}
u_{1}(i)+\frac{1}{24}\left(19 u_{1}^{\prime}(i)-5 u_{1}^{\prime}(i-1)+u_{1}^{\prime}(i-2)\right) \\
u_{2}(i)+\frac{1}{24}\left(19 u_{2}^{\prime}(i)-5 u_{2}^{\prime}(i-1)+u_{2}^{\prime}(i-2)\right)
\end{array}\right) .
\end{aligned}
$$

See [30] for a detailed discussion.

For inward integration, the 4th order Adams inward extrapolation is given by

$$
y_{i-1}=y_{i}-\frac{1}{24}\left(55 y_{i}^{\prime}-59 y_{i+1}^{\prime}+37 y_{i+2}^{\prime}-9 y_{i+3}^{\prime}\right)
$$

and interpolation by

$$
y_{i-1}=y_{i}-\frac{1}{24}\left(9 y_{i-1}^{\prime}+19 y_{i}^{\prime}-5 y_{i+1}^{\prime}+y_{i+2}^{\prime}\right),
$$


in which case, analytic interpolation gives:

$$
\begin{aligned}
\lambda & =-\frac{9}{24}, \\
\Delta & =1+\lambda^{2} R^{\prime 2}(i-1) \operatorname{det} C(i-1), \\
M & =\frac{1}{\Delta}\left(\left(\begin{array}{ll}
1 & 0 \\
0 & 1
\end{array}\right)+\lambda R^{\prime}(i-1)\left(\begin{array}{cc}
-C_{22}(i-1) & C_{12}(i-1) \\
C_{21}(i-1)-C_{11}(i-1)
\end{array}\right)\right), \\
\left(\begin{array}{l}
u_{1}(i-1) \\
u_{2}(i-1)
\end{array}\right) & =M\left(\begin{array}{l}
u_{1}(i)-\frac{1}{24}\left(19 u_{1}^{\prime}(i)-5 u_{1}^{\prime}(i+1)+u_{1}^{\prime}(i+2)\right) \\
u_{2}(i)-\frac{1}{24}\left(19 u_{2}^{\prime}(i)-5 u_{2}^{\prime}(i+1)+u_{2}^{\prime}(i+2)\right)
\end{array}\right) .
\end{aligned}
$$

In practice, we use RK4 to compute the first 4 points needed by implicit Adams, then switch to the more accurate implicit Adams method to compute the remaining points.

\subsection{Mesh}

The methods discussed here can use any desired mesh: uniform, hyperbolic, exponential, or other. In practice, however, as we show below, most atomic structure codes use some form of exponential (or "logarithmic") mesh, as this gives an efficient concentration of mesh points in the vicinity of the Coulomb singularity, where wavefunctions, densities, and potentials vary most rapidly. Given their particular prevalence, we discuss exponential meshes below, and provide associated routines in the dftatom distribution.

Every exponential mesh can be written as a function of exactly four parameters, $r_{\min }, r_{\max }, a$, and $N$, as follows:

$$
\begin{aligned}
R_{i} & =\alpha\left(e^{\beta(i-1)}-1\right)+r_{\min }, \\
\alpha & =\frac{r_{\max }-r_{\min }}{e^{\beta N}-1} \\
\beta & =\frac{\log a}{N-1} .
\end{aligned}
$$

for $i=1,2, \ldots, N+1$. Note that in the above we have explicitly included an arbitrary shift of origin. Without this, three parameters are of course sufficient to specify an exponential mesh. As we elaborate below, however, the flexibility to choose the initial point independent of other mesh parameters affords additional efficiency when employing asymptotic expressions for initial values. In the above form, all parameters have direct physical meaning: the mesh starts at $R_{1}=r_{\min }$ and ends at $R_{N+1}=r_{\max }$; as shown below, the parameter $a$ is the ratio of rightmost to leftmost element (mesh interval) lengths 
(determining the mesh gradation), and $N$ is the number of elements in the mesh. The ratio $q$ of any two successive elements can be calculated from (89):

$$
q=\frac{R_{n+1}-R_{n}}{R_{n}-R_{n-1}}=e^{\beta}=a^{\frac{1}{N-1}} .
$$

It follows that the mesh gradation $a$ can be expressed using the ratio $q$ as $a=q^{N-1}$ and thus $a$ is the ratio of rightmost to leftmost element lengths.

The main advantage of this parametrization is that $r_{\min }$ and $r_{\max }$ are decoupled from the mesh gradation and number of elements and can thus be varied independently. Once $r_{\min }$ is determined to provide sufficiently accurate asymptotic values, there is still freedom to change the gradation of the mesh by changing $a$. Then, after determining the optimal $a$, mesh convergence can be achieved by simply increasing $N$. By having the option to optimize $a$ independent of both $r_{\min }$ and $N$, one can reduce the number of elements $N$ required for a given accuracy, thus speeding up the calculation.

Exponential meshes in common use are special cases of the above more general one. For example the first and second meshes in [27] are given by

$$
r_{i}=r_{\min }\left(\frac{r_{\max }}{r_{\min }}\right)^{(i-1) / N} .
$$

Here, the meaning of $i, N, r_{\min }$, and $r_{\max }$ is the same as in (89). This form is a special case of $(89)$ with $a=\left(r_{\max } / r_{\min }\right)^{\frac{N-1}{N}}$. As such, the mesh is determined by three parameters $r_{\min }, r_{\max }$, and $N$, and the mesh gradation $a$ depends on $N$ and the fraction $r_{\max } / r_{\min }$. Note that this mesh does not allow $r_{\min }=0$ (the mesh gradation would become infinite). For uranium, the first mesh has $N=15788, r_{\min }=\frac{1}{160 \times 92}$, and $r_{\max }=50$; from which it follows that $a=$ $7.353705 \times 10^{5}$. The second mesh has $N=8000, r_{\min }=\frac{10^{-6}}{92}$, and $r_{\max }=\frac{800}{\sqrt{92}}$; from which it follows $a=7.651530 \times 10^{9}$.

The third mesh in [27] is given by

$$
r_{i}=A\left(e^{B i}-1\right)
$$

The meaning of $i$ is the same as in (89). The mesh is determined by three parameters $A, B$, and $N$. This is a special case of (89) with $r_{\min }=A\left(e^{B}-1\right)$, $r_{\max }=A\left(e^{B(N+1)}-1\right)$, and $a=e^{B(N-1)}$. For uranium, the values in [27] are $A=\frac{4.34}{92} \times 10^{-6}, B=0.002304$, and $N=9019$; from which it follows that $r_{\min }=1.088140 \times 10^{-10}, r_{\max }=50.031620$, and $a=1.055702 \times 10^{9}$.

The fourth mesh in [27] is given by:

$$
\rho_{i}=\log R_{i}
$$


Table 2

\begin{tabular}{ccccc} 
& $r_{\min }$ & $r_{\max }$ & $a$ & $N$ \\
\hline LDA & $1.0 \mathrm{e}-07$ & 50.0 & $2.7 \mathrm{e}+06$ & 4866 \\
RLDA & $1.0 \mathrm{e}-08$ & 50.0 & $6.2 \mathrm{e}+07$ & 5268
\end{tabular}

Optimal mesh parameters for $10^{-6}$ a.u. accuracy in total energy of uranium.

where $\rho_{i}$ is a uniform mesh with $N$ elements such that $R_{1}=r_{\min }$ and $R_{N+1}=$ $r_{\max }$. It follows that $\rho_{i}=\frac{i-1}{N} \log \frac{r_{\max }}{r_{\min }}+\log r_{\min }$. Then substituting into (95) and simplifying, the expression (93) is obtained. As such, the fourth mesh is equivalent to the first. For uranium, the values in [27] are $N=2837, r_{\text {min }}=$ $\frac{10^{-6}}{92}$, and $r_{\max }=50$; from which it follows that $a=4.564065 \times 10^{9}$.

To summarize, the parameters of the four meshes for uranium in [27] are then as follows.

\begin{tabular}{|c|c|c|c|c|}
\hline & r_min & r_max & a & $\mathrm{N}$ \\
\hline 1 & $6.79347826087 e-05$ & 50.0 & $7.353705 e+05$ & 15788 \\
\hline 2 & $1.08695652174 \mathrm{e}-08$ & 83.4057656228 & $7.651530 e+09$ & 8000 \\
\hline 3 & $1.08814001246 e-10$ & 50.0316203306 & $1.055702 \mathrm{e}+09$ & 9019 \\
\hline 4 & $1.08695652174 \mathrm{e}-08$ & 50.0 & $4.564065 e+09$ & 2837 \\
\hline
\end{tabular}

In Section 4.4, we determine optimal parameters for LDA and RLDA calculations, as shown in Table 2. Here, the given $r_{\min }, r_{\max }$, and $a$ can be used for all atoms, while the given $N$ is sufficient for $10^{-6}$ a.u. accuracy in total energy for uranium; it can be increased for higher accuracy and decreased for lower atomic numbers $Z$ to improve speed while retaining $10^{-6}$ accuracy, as we show in Section 4.4.

\subsection{Shooting method for Schrödinger \&3 Dirac eigenproblems}

The required eigenfunctions are determined by the shooting method [18]: i.e., eigenvalues are guessed, radial integrations are performed, guesses are updated, and the process is repeated until convergence is achieved to the desired tolerance. For efficiency, we employ a combination of inward and outward integrations, bisection for robustness, and perturbation theory for efficiency once the solution is sufficiently close to convergence.

The outward integration starts at $r_{\text {min }}$ using the asymptotic from Section 2 with $r=r_{\min }$, then RK4 is used for the first four steps, then Adams for the rest. For the inward integration, a starting point $r_{m}$ is determined such that $e^{-\lambda r_{m}}$ is on the order of machine epsilon (e.g., $\sim 10^{-16}$ in double precision), with $\lambda$ determined from the large- $r$ asymptotic for the Schrödinger or Dirac 
equation, as appropriate (Section 2). Then $e^{-\lambda r_{m}}$ is used for the last four points up to $r_{m}$ and inward Adams is used for the rest.

The outward integration is performed up to the classical turning point $r_{\text {ctp }}$, defined by $V\left(r_{\text {ctp }}\right)=E$. Bisection is used to converge the energy to the point that the associated radial solution has the correct number of nodes $(n-l-1)$. After the correct number of nodes is obtained, two approaches are considered to complete the eigenfunction computation.

The first approach is bisection: the outward integration is continued from $r_{\text {ctp }}$ to the rest of the domain and the total number of nodes is counted. If the number is strictly greater than $n-l-1$, then the energy $E$ is above the eigenvalue, otherwise it is below the eigenvalue. Bisection is used to refine the eigenvalue to specified accuracy.

The second approach uses a perturbation correction for the energy, using both inward and outward integrations. It typically converges an order of magnitude faster than outward-only bisection once the correct number of nodes is obtained. For the Schrödinger equation, the following correction is used [30,3739]:

$$
E_{2} \approx E_{1}+\frac{P\left(r_{\text {ctp }}\right)\left(Q\left(r_{\text {ctp }}^{-}\right)-Q\left(r_{\text {ctp }}^{+}\right)\right)}{2 \int_{0}^{\infty} P^{2}(r) \mathrm{d} r},
$$

where $E_{1}$ is the energy used to calculate $P(r)$ and $Q(r)$. The inward integration is multiplied by a constant so that it matches (in value) the outward integration at the classical turning point $r_{\text {ctp }}$. This leaves a jump in the derivative of the outward integration $Q\left(r_{\text {ctp }}^{-}\right)$and inward integration $Q\left(r_{\text {ctp }}^{+}\right) . E_{2}$ is the new energy for the next iteration. Usually around five iterations are sufficient to achieve $10^{-13}$ accuracy in energy.

For Dirac equation, the following correction is used [30,40]:

$$
E_{2} \approx E_{1}+c \frac{P\left(r_{\text {ctp }}\right)\left(Q\left(r_{\text {ctp }}^{-}\right)-Q\left(r_{\text {ctp }}^{+}\right)\right)}{\int_{0}^{\infty}\left(P^{2}(r)+Q^{2}(r)\right) \mathrm{d} r} .
$$

After the correct number of nodes is determined, the perturbation correction is tried first. If it fails to converge (as can occur, e.g., for a too-confined domain), we fall back to outward-only bisection for robustness. For positive energies, the perturbation correction is inapplicable and outward-only bisection is used exclusively. Physically, the outward-only bisection method solves the atom in an infinite potential well of the size of the domain while the inward-outward perturbation method solves an atom in an infinite domain (by virtue of the asymptotic inward integration). As such, for large $r_{\max }$, the two methods converge to the same value, but for small $r_{\max }$ the eigenvalues can differ between methods. (An $r_{\max }$ study is needed to determine sufficient $r_{\max }$, as we show in Section 4). 
All normalization and other integrals are calculated on $\left[r_{\min }, r_{\max }\right]$, avoiding the Coulomb singularity at $r=0$. Hence, convergence with respect to $r_{\text {min }}$ (typically $\sim 10^{-8}$ a.u.) must be checked, as we show in Section 4 .

\subsection{Outward Poisson integration}

Since asymptotics are known at both ends of the domain (Section 2.3), it is possible to integrate the Poisson equation either inward or outward. Unlike other available codes [10-16], we employ outward integration here in order to more accurately resolve rapid variations in core region. This better resolves the most tightly bound states (e.g., $1 s$ and $2 s$ ), in particular.

Since charge is omitted around the Coulomb singularity at $r=0(0<r<$ $\left.r_{\text {min }}\right)$, we take as initial conditions, consistent with Eqs. (42) and (43):

$$
\begin{aligned}
& V_{H}\left(r_{\min }\right)=4 \pi \int_{r_{\min }}^{r_{\max }} r n(r) \mathrm{d} r, \\
& V_{H}^{\prime}\left(r_{\min }\right)=0 .
\end{aligned}
$$

Then RK4 is used for the first four points, after which the Adams method is used with predictor-corrector for the rest. Here again, convergence with respect to $r_{\min }$ must be checked, as we show in Section 4 .

Having a precise Poisson solver is crucial to resolve all Kohn-Sham states accurately. With this outward solver, Kohn-Sham eigenvalues are typically an order of magnitude more precise than the total energy, with the most pronounced effect on the more tightly bound states. This is in contrast to other solvers [27] which typically resolve eigenvalues less accurately than total energies.

Outward integration of the Poisson equation has been considered previously $[41,42]$. However, rather than solving the differential equation, these authors compute $V_{H}(r)$ using the integral formulation, which has implicit the initial conditions $V_{H}\left(r_{\min }\right)=V_{H}^{\prime}\left(r_{\min }\right)=0$, shifting energies by $4 \pi \int_{r_{\min }}^{r_{\max }} r n(r) \mathrm{d} r$, subtracted subsequently. Outward integration of the differential equation has been discussed in [39], where integration of the differential equation for $Y(r)=$ $r V_{H}(r)$ is considered with initial conditions $Y(r)=Y^{\prime}(r)=0$, with subtraction of the associated energy shift subsequently. 


\section{Results}

\subsection{Analytic test case: $V=-Z / r$}

To verify the accuracy of the solver and establish convergence indicators, the Schrödinger and Dirac equations are solved for potential $V=-Z / r$ with $Z=92$, for which analytic results are available. $r_{\min }, r_{\max }$, and $N$ convergence studies are run for all eigenvalues with $n \leq 7$. In the resulting plots, $E-E_{\text {prev }}$ gives the change in energy with change of parameter being studied: e.g., as $r_{\min }$ is decreased toward convergence, $r_{\min }=10^{-4}, 10^{-5}, \ldots, 10^{-14}, E-E_{\text {prev }}$ at $r_{\min }=10^{-7}$ is the difference of computed energies at $r_{\min }=10^{-7}$ and $r_{\min }=10^{-6} . E-E_{\text {conv }}$ is the difference of the computed energy from the fully converged value: e.g., $E-E_{\text {conv }}$ at $r_{\min }=10^{-7}$ is the difference of energy at $r_{\text {min }}=10^{-7}$ from the fully converged value at $r_{\min }$ sufficiently small that reducing it further does not reduce the error further (due to finite precision). Note that convergence plots omit points at converged values of parameters $\left(r_{\min }, r_{\max }, N\right)$ because energy differences $E-E_{\text {conv }}$ are identically zero there, and so cannot be plotted on the log scale.

For the Schrödinger equation, the $r_{\min }$ convergence study was run with $a=$ $10^{9}, r_{\max }=50$, and $N=50000$. From the resulting plot (Fig. 1a), we see that $r_{\min }=10^{-7}$ is a converged value; decreasing $r_{\min }$ further does not reduce energy differences further. The $r_{\max }$ convergence study was run with $a=$ $2.7 \cdot 10^{6}, r_{\min }=10^{-7}$, and $N=50000$. From the resulting plot (Fig. 1b), we see that $r_{\max }=3.5$ is a converged value. Finally, the $N$ study was run with $a=2.7 \cdot 10^{6}, r_{\min }=10^{-7}$, and $r_{\max }=50$. From the plot (Fig. 1c), it is determined that $N=50000$ is a converged value.

From these results, it is seen that the fixed parameters for each convergence study were fully converged and the error indicator in each case was below $10^{-10}$ a.u. Table 3 compares the computed eigenvalues to exact values $\left(-\frac{Z^{2}}{2 n^{2}}\right)$ for this analytic test case and it is verified that the absolute error of the computed values is below $10^{-10}$, as indicated by the convergence studies.

Of course, smaller $N$ could be used to achieve the above accuracies using the converged $r_{\min }$ and $r_{\max }$ values indicated by the above studies, then optimizing the mesh gradation $a$ to minimize the required $N$. The purpose of the present analytic case study, however, is to verify the accuracy of the solver and establish the robustness of convergence indicators, $E-E_{\text {prev }}$ and $E-E_{\text {conv }}$. Optimal $a$ and $N$ are discussed in Section 4.4 .

For the Dirac equation, the $N$ study is first done for $r_{\min }$ larger than $10^{-11}$ and $N=200000$ was obtained as a converged value $\left(a=10^{9}\right.$ was used so that convergence was obtained for tractable $N$ for all $\left.r_{\min }\right)$. The $r_{\min }$ study was 


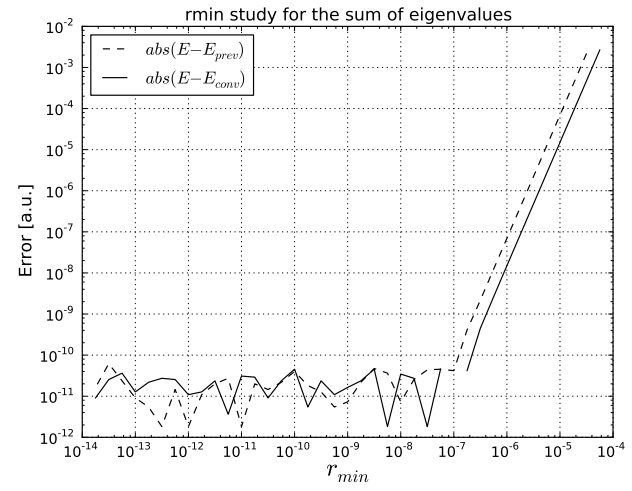

(a) $r_{\min }$ study

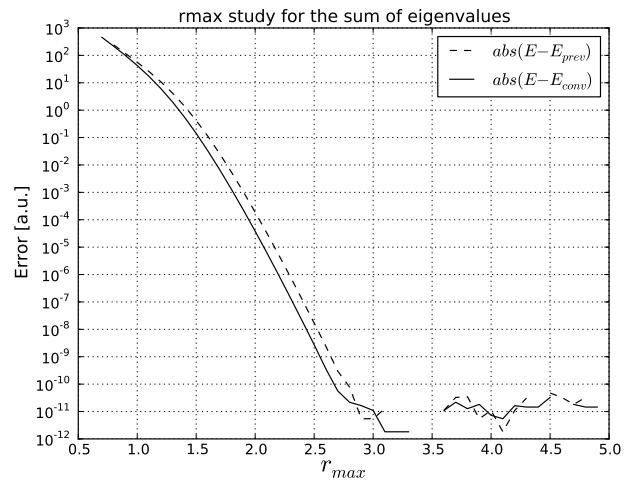

(b) $r_{\max }$ study

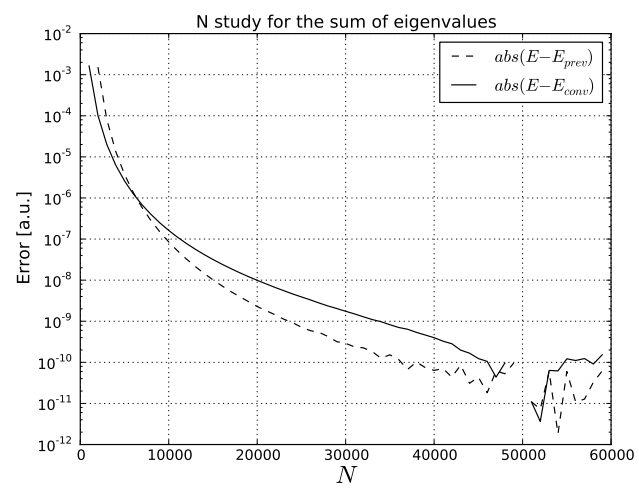

(c) $N$ study

Fig. 1. Convergence studies for the sum of Schrödinger eigenvalues for $V=-Z / r$.

then run and determined $r_{\min }=10^{-8}$ as a converged value, giving accuracy better than $5 \cdot 10^{-10}$ a.u. $r_{\max }=50$ was used and it was verified that it is a converged value. Table 4 shows a comparison of computed and exact Dirac 
Table 3

\begin{tabular}{ccccc}
$\mathrm{n}$ & 1 & dftatom & exact & diff \\
\hline 1 & 0 & -4232.00000000001 & -4232.00000000000 & $9.09 \mathrm{e}-12$ \\
2 & 0 & -1058.00000000001 & -1058.00000000000 & $7.28 \mathrm{e}-12$ \\
2 & 1 & -1058.00000000000 & -1058.00000000000 & $9.09 \mathrm{e}-13$ \\
3 & 0 & -470.222222222232 & -470.222222222222 & $9.55 \mathrm{e}-12$ \\
3 & 1 & -470.222222222224 & -470.222222222222 & $2.22 \mathrm{e}-12$ \\
3 & 2 & -470.222222222224 & -470.222222222222 & $2.22 \mathrm{e}-12$ \\
4 & 0 & -264.500000000013 & -264.500000000000 & $1.33 \mathrm{e}-11$ \\
4 & 1 & -264.500000000005 & -264.500000000000 & $5.00 \mathrm{e}-12$ \\
4 & 2 & -264.500000000005 & -264.500000000000 & $5.00 \mathrm{e}-12$ \\
4 & 3 & -264.499999999997 & -264.500000000000 & $3.18 \mathrm{e}-12$ \\
5 & 0 & -169.280000000016 & -169.280000000000 & $1.61 \mathrm{e}-11$ \\
5 & 1 & -169.280000000011 & -169.280000000000 & $1.08 \mathrm{e}-11$ \\
5 & 2 & -169.280000000006 & -169.280000000000 & $5.57 \mathrm{e}-12$ \\
5 & 3 & -169.280000000006 & -169.280000000000 & $5.57 \mathrm{e}-12$ \\
5 & 4 & -169.280000000000 & -169.280000000000 & $3.13 \mathrm{e}-13$ \\
6 & 0 & -117.555555555579 & -117.555555555556 & $2.34 \mathrm{e}-11$ \\
6 & 1 & -117.555555555579 & -117.555555555556 & $2.34 \mathrm{e}-11$ \\
6 & 2 & -117.555555555572 & -117.555555555556 & $1.61 \mathrm{e}-11$ \\
6 & 3 & -117.555555555564 & -117.555555555556 & $8.84 \mathrm{e}-12$ \\
6 & 4 & -117.555555555557 & -117.555555555556 & $1.55 \mathrm{e}-12$ \\
6 & 5 & -117.555555555557 & -117.555555555556 & $1.55 \mathrm{e}-12$ \\
7 & 0 & -86.3673469388100 & -86.3673469387755 & $3.44 \mathrm{e}-11$ \\
7 & 1 & -86.3673469388046 & -86.3673469387755 & $2.91 \mathrm{e}-11$ \\
7 & 2 & -86.3673469387993 & -86.3673469387755 & $2.38 \mathrm{e}-11$ \\
7 & 3 & -86.3673469387886 & -86.3673469387755 & $1.31 \mathrm{e}-11$ \\
7 & 4 & -86.3673469387832 & -86.3673469387755 & $7.73 \mathrm{e}-12$ \\
7 & 5 & -86.3673469387779 & -86.3673469387755 & $2.36 \mathrm{e}-12$ \\
7 & 6 & -86.3673469387779 & -86.3673469387755 & $2.36 \mathrm{e}-12$
\end{tabular}

Computed and exact Schrödinger eigenvalues for $V=-\frac{Z}{r}$.

eigenvalues

$$
\begin{aligned}
E_{n \kappa} & =\frac{c^{2}}{\sqrt{1+\frac{(Z / c)^{2}}{(n-|\kappa|+\beta)^{2}}}}-c^{2}, \\
\beta & =\sqrt{\kappa^{2}-(Z / c)^{2}}
\end{aligned}
$$

for the Coulomb problem, and it is verified that the absolute error is below $5 \cdot 10^{-10}$ a.u., as indicated by the convergence studies. Note: all numerical and analytic calculations in this paper use the 1986 CODATA [43] recommended value $c=137.0359895$ a.u., the same value as in NIST benchmarks [27].

\subsection{Nonsingular test case: $V=\frac{1}{2} \omega^{2} r^{2}$}

Table 5 shows computed Schrödinger eigenvalues for the harmonic oscillator potential $V(r)=\frac{1}{2} \omega^{2} r^{2}$ with $\omega=1$ compared to exact values

$$
E_{n l}=\omega\left(2 n-l-\frac{1}{2}\right)
$$




\begin{tabular}{|c|c|c|c|c|c|}
\hline $\mathrm{n}$ & l & s & dftatom & exact & diff \\
\hline 1 & 0 & 1 & -4861.19802311923 & -4861.19802311937 & $1.41 \mathrm{e}-10$ \\
\hline 2 & 0 & 1 & -1257.39589025788 & -1257.39589025789 & $8.41 \mathrm{e}-12$ \\
\hline 2 & 1 & 1 & -1089.61142091988 & -1089.61142091987 & $1.59 \mathrm{e}-12$ \\
\hline 2 & 1 & -1 & -1257.39589025790 & -1257.39589025789 & $1.75 \mathrm{e}-11$ \\
\hline 3 & 0 & 1 & -539.093341793886 & -539.093341793890 & $3.98 \mathrm{e}-12$ \\
\hline 3 & 1 & 1 & -489.037087678200 & -489.037087678200 & $1.14 \mathrm{e}-13$ \\
\hline 3 & 1 & -1 & -539.093341793896 & -539.093341793890 & $5.68 \mathrm{e}-12$ \\
\hline 3 & 2 & 1 & -476.261595161156 & -476.261595161155 & $1.36 \mathrm{e}-12$ \\
\hline 3 & 2 & -1 & -489.037087678199 & -489.037087678200 & $6.25 \mathrm{e}-13$ \\
\hline 4 & 0 & 1 & -295.257844100398 & -295.257844100397 & $1.42 \mathrm{e}-12$ \\
\hline 4 & 1 & 1 & -274.407758840063 & -274.407758840065 & $2.50 \mathrm{e}-12$ \\
\hline 4 & 1 & -1 & -295.257844100398 & -295.257844100397 & $1.25 \mathrm{e}-12$ \\
\hline 4 & 2 & 1 & -268.965877827131 & -268.965877827130 & $9.66 \mathrm{e}-13$ \\
\hline 4 & 2 & -1 & -274.407758840063 & -274.407758840065 & $1.88 \mathrm{e}-12$ \\
\hline 4 & 3 & 1 & -266.389447187815 & -266.389447187816 & $3.41 \mathrm{e}-13$ \\
\hline 4 & 3 & -1 & -268.965877827131 & -268.965877827130 & $1.53 \mathrm{e}-12$ \\
\hline 5 & 0 & 1 & -185.485191678549 & -185.485191678552 & $3.01 \mathrm{e}-12$ \\
\hline 5 & 1 & 1 & -174.944613583463 & -174.944613583462 & $7.67 \mathrm{e}-13$ \\
\hline 5 & 1 & -1 & -185.485191678550 & -185.485191678552 & $1.79 \mathrm{e}-12$ \\
\hline 5 & 2 & 1 & -172.155252323734 & -172.155252323737 & $2.67 \mathrm{e}-12$ \\
\hline 5 & 2 & -1 & -174.944613583463 & -174.944613583462 & $6.54 \mathrm{e}-13$ \\
\hline 5 & 3 & 1 & -170.828937049879 & -170.828937049879 & $1.14 \mathrm{e}-13$ \\
\hline 5 & 3 & -1 & -172.155252323735 & -172.155252323737 & $1.88 \mathrm{e}-12$ \\
\hline 5 & 4 & 1 & -170.049934288550 & -170.049934288552 & $2.36 \mathrm{e}-12$ \\
\hline 5 & 4 & -1 & -170.828937049878 & -170.828937049879 & $1.19 \mathrm{e}-12$ \\
\hline 6 & 0 & 1 & -127.093638842628 & -127.093638842631 & $2.80 \mathrm{e}-12$ \\
\hline 6 & 1 & 1 & -121.057538029547 & -121.057538029549 & $1.44 \mathrm{e}-12$ \\
\hline 6 & 1 & -1 & -127.093638842628 & -127.093638842631 & $2.26 \mathrm{e}-12$ \\
\hline 6 & 2 & 1 & -119.445271987140 & -119.445271987141 & $6.39 \mathrm{e}-13$ \\
\hline 6 & 2 & -1 & -121.057538029547 & -121.057538029549 & $1.38 \mathrm{e}-12$ \\
\hline 6 & 3 & 1 & -118.676410324351 & -118.676410324351 & $1.28 \mathrm{e}-13$ \\
\hline 6 & 3 & -1 & -119.445271987140 & -119.445271987141 & $3.13 \mathrm{e}-13$ \\
\hline 6 & 4 & 1 & -118.224144624903 & -118.224144624903 & $2.42 \mathrm{e}-13$ \\
\hline 6 & 4 & -1 & -118.676410324351 & -118.676410324351 & $1.71 \mathrm{e}-13$ \\
\hline 6 & 5 & 1 & -117.925825597294 & -117.925825597293 & $1.34 \mathrm{e}-12$ \\
\hline 6 & 5 & -1 & -118.224144624903 & -118.224144624903 & $4.41 \mathrm{e}-13$ \\
\hline 7 & 0 & 1 & -92.4407876009401 & -92.4407876009427 & $2.59 \mathrm{e}-12$ \\
\hline 7 & 1 & 1 & -88.6717490520179 & -88.6717490520168 & $1.05 \mathrm{e}-12$ \\
\hline 7 & 1 & -1 & -92.4407876009404 & -92.4407876009427 & $2.30 \mathrm{e}-12$ \\
\hline 7 & 2 & 1 & -87.6582876318895 & -87.6582876318935 & $3.94 \mathrm{e}-12$ \\
\hline 7 & 2 & -1 & -88.6717490520179 & -88.6717490520168 & $1.08 \mathrm{e}-12$ \\
\hline 7 & 3 & 1 & -87.1739666719491 & -87.1739666719477 & $1.35 \mathrm{e}-12$ \\
\hline 7 & 3 & -1 & -87.6582876318897 & -87.6582876318935 & $3.77 \mathrm{e}-12$ \\
\hline 7 & 4 & 1 & -86.8887663909435 & -86.8887663909409 & $2.64 \mathrm{e}-12$ \\
\hline 7 & 4 & -1 & -87.1739666719491 & -87.1739666719477 & $1.38 \mathrm{e}-12$ \\
\hline 7 & 5 & 1 & -86.7005195728073 & -86.7005195728088 & $1.55 \mathrm{e}-12$ \\
\hline 7 & 5 & -1 & -86.8887663909439 & -86.8887663909409 & $3.06 \mathrm{e}-12$ \\
\hline 7 & 6 & 1 & -86.5668751023587 & -86.5668751023550 & $3.77 \mathrm{e}-12$ \\
\hline 7 & 6 & -1 & -86.7005195728072 & -86.7005195728088 & $1.63 \mathrm{e}-12$ \\
\hline
\end{tabular}

Table 4

Computed and exact Dirac eigenvalues for $V=-\frac{Z}{r}$.

Asymptotics (10), (11) are used for outward integration, with perturbation correction turned off due to positive energies. It is seen that $r_{\min }=10^{-7}$ a.u., $r_{\max }=10$ a.u., $a=10$ and $N=5000$ are sufficient to obtain eigenvalues accurate to $<10^{-8}$ a.u.

Table 6 shows Dirac eigenvalues for the harmonic oscillator potential $V(r)=$ 
Table 5

\begin{tabular}{ccccc}
$\mathrm{n}$ & 1 & dftatom & exact & diff \\
\hline 1 & 0 & 1.500000000168 & 1.500000000000 & $1.68 \mathrm{E}-10$ \\
2 & 0 & 3.500000000587 & 3.500000000000 & $5.87 \mathrm{E}-10$ \\
2 & 1 & 2.499999999999 & 2.500000000000 & $8.53 \mathrm{E}-13$ \\
3 & 0 & 5.500000001140 & 5.500000000000 & $1.14 \mathrm{E}-09$ \\
3 & 1 & 4.499999999993 & 4.500000000000 & $7.22 \mathrm{E}-12$ \\
3 & 2 & 3.499999999999 & 3.500000000000 & $1.19 \mathrm{E}-12$ \\
4 & 0 & 7.500000001777 & 7.500000000000 & $1.78 \mathrm{E}-09$ \\
4 & 1 & 6.499999999968 & 6.500000000000 & $3.18 \mathrm{E}-11$ \\
4 & 2 & 5.499999999990 & 5.500000000000 & $1.04 \mathrm{E}-11$ \\
4 & 3 & 4.499999999998 & 4.500000000000 & $1.53 \mathrm{E}-12$ \\
5 & 0 & 9.500000002451 & 9.500000000000 & $2.45 \mathrm{E}-09$ \\
5 & 1 & 8.499999999900 & 8.500000000000 & $1.00 \mathrm{E}-10$ \\
5 & 2 & 7.499999999955 & 7.500000000000 & $4.52 \mathrm{E}-11$ \\
5 & 3 & 6.499999999986 & 6.500000000000 & $1.42 \mathrm{E}-11$ \\
5 & 4 & 5.499999999998 & 5.500000000000 & $2.44 \mathrm{E}-12$ \\
6 & 0 & 11.500000003104 & 11.500000000000 & $3.10 \mathrm{E}-09$ \\
6 & 1 & 10.499999999751 & 10.500000000000 & $2.49 \mathrm{E}-10$ \\
6 & 2 & 9.499999999864 & 9.500000000000 & $1.36 \mathrm{E}-10$ \\
6 & 3 & 8.499999999940 & 8.500000000000 & $6.03 \mathrm{E}-11$ \\
6 & 4 & 7.499999999982 & 7.500000000000 & $1.85 \mathrm{E}-11$ \\
6 & 5 & 6.499999999997 & 6.500000000000 & $2.79 \mathrm{E}-12$ \\
7 & 0 & 13.500000003665 & 13.500000000000 & $3.66 \mathrm{E}-09$ \\
7 & 1 & 12.499999999466 & 12.500000000000 & $5.34 \mathrm{E}-10$ \\
7 & 2 & 11.499999999672 & 11.500000000000 & $3.28 \mathrm{E}-10$ \\
7 & 3 & 10.499999999824 & 10.500000000000 & $1.76 \mathrm{E}-10$ \\
7 & 4 & 9.499999999923 & 9.500000000000 & $7.66 \mathrm{E}-11$ \\
7 & 5 & 8.499999999977 & 8.500000000000 & $2.34 \mathrm{E}-11$ \\
7 & 6 & 7.499999999996 & 7.500000000000 & $3.69 \mathrm{E}-12$ \\
& & & &
\end{tabular}

Computed and exact Schrödinger eigenvalues for $V=\frac{1}{2} r^{2}$.

$\frac{1}{2} \omega^{2} r^{2}$ with $\omega=1$. Asymptotics (24), (25) and (26), (27) are used for outward integration, with perturbation correction again turned off due to positive energies. Lacking analytic results for comparison, we compared instead to independent high-order finite-element based calculations [44] which require no assumptions of asymptotic forms or determination of sufficiently small $r_{\min }>0$. For $r_{\min }=10^{-8}$ a.u., $r_{\max }=10$ a.u., $a=80$, and $N=5000$, we find agreement of all eigenvalues to $<10^{-8}$ a.u., with values very near to the corresponding Schrödinger results due to the relative weakness of the potential, in stark contrast to the singular Coulombic case. Note that, unlike the case of a singular potential, the number of nodes for $\kappa>0$ is only $n-l$, whereas the number of nodes for $\kappa<0$ remains $n-l-1$, as in the singular case.

\subsection{Double minimum potential}

A problem that is known to cause difficulties for shooting solvers is the double minimum problem. A classical example is the electronically excited $E$, $F^{1} \Sigma_{g}^{+}$state of the hydrogen molecule $[45,46,20]$, but such potentials occur in electronic structure also [47].

The potential is given on a discrete grid in Table I of Ref. [45]. There are 
Table 6

\begin{tabular}{|c|c|c|c|c|c|c|c|}
\hline $\mathrm{n}$ & 1 & $\mathrm{~s}$ & dftatom & $\mathrm{n}$ & 1 & $\mathrm{~s}$ & dftatom \\
\hline 1 & 0 & 0 & 1.49999501 & 6 & 0 & 0 & 11.49869739 \\
\hline 2 & 0 & 0 & 3.49989517 & 6 & 1 & 0 & 10.49893692 \\
\hline 2 & 1 & 0 & 2.49997504 & 6 & 1 & 1 & 10.49889699 \\
\hline 2 & 1 & 1 & 2.49993510 & 6 & 2 & 0 & 9.49916315 \\
\hline 3 & 0 & 0 & 5.49971548 & 6 & 2 & 1 & 9.49909661 \\
\hline 3 & 1 & 0 & 4.49983527 & 6 & 3 & 0 & 8.49937608 \\
\hline 3 & 1 & 1 & 4.49979534 & 6 & 3 & 1 & 8.49928292 \\
\hline 3 & 2 & 0 & 3.49994176 & 6 & 4 & 0 & 7.49957572 \\
\hline 3 & 2 & 1 & 3.49987520 & 6 & 4 & 1 & 7.49945594 \\
\hline 4 & 0 & 0 & 7.49945594 & 6 & 5 & 0 & 6.49976205 \\
\hline 4 & 1 & 0 & 6.49961565 & 6 & 5 & 1 & 6.49961565 \\
\hline 4 & 1 & 1 & 6.49957572 & 7 & 0 & 0 & 13.49819839 \\
\hline 4 & 2 & 0 & 5.49976206 & 7 & 1 & 0 & 12.49847782 \\
\hline 4 & 2 & 1 & 5.49969551 & 7 & 1 & 1 & 12.49843790 \\
\hline 4 & 3 & 0 & 4.49989517 & 7 & 2 & 0 & 11.49874396 \\
\hline 4 & 3 & 1 & 4.49980199 & 7 & 2 & 1 & 11.49867742 \\
\hline 5 & 0 & 0 & 9.49911657 & 7 & 3 & 0 & 10.49899680 \\
\hline 5 & 1 & 0 & 8.49931620 & 7 & 3 & 1 & 10.49890364 \\
\hline 5 & 1 & 1 & 8.49927627 & 7 & 4 & 0 & 9.49923634 \\
\hline 5 & 2 & 0 & $\begin{array}{l}7.49950252 \\
7.49943598\end{array}$ & 7 & 4 & 1 & 9.49911657 \\
\hline 5 & 2 & 1 & $\begin{array}{l}7.49943598 \\
6.49967554\end{array}$ & 7 & 5 & 0 & 8.49946258 \\
\hline 5 & 3 & 0 & $\begin{array}{l}6.49967554 \\
6.49958238\end{array}$ & 7 & 5 & 1 & 8.49931619 \\
\hline 5 & 4 & $\begin{array}{l}1 \\
0\end{array}$ & 5.499002000 & 7 & 6 & 0 & 7.49967553 \\
\hline 5 & 4 & 1 & 5.49971547 & 7 & 6 & 1 & 7.49950251 \\
\hline
\end{tabular}

Computed Dirac eigenvalues for $V=\frac{1}{2} r^{2}$.

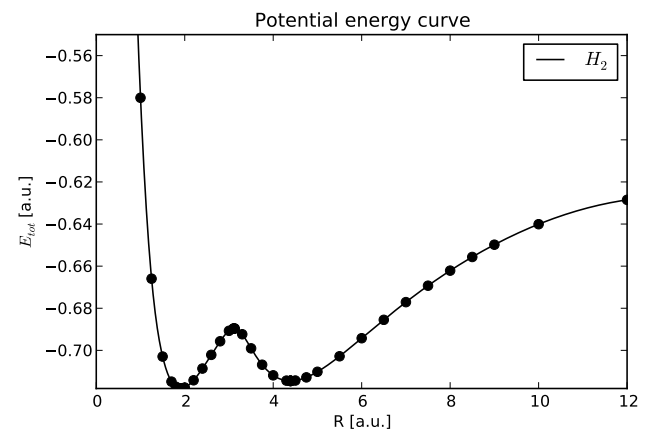

Fig. 2. Double minimum potential discrete values (dots) and cubic spline interpolation (line).

several options for interpolating the potential, each giving slightly different energies. For the present purposes, cubic spline interpolation was used, with consistent boundary conditions at both ends (second derivative consistent with cubic interpolant), see Fig. 2. We have implemented Lagrange (as in [46]) and Hermite (as in [45]) interpolation also, included in the distribution of dftatom $[29]$.

The problem is solved for $r_{\max }=12$ a.u., with reduced mass $\mu=1836.12 / 2$ a.u. and conversion of $E$ from a.u. to $\mathrm{cm}^{-1}$ via $(-0.625-E) \cdot 219474.62$, as in [45]. First, an $N$-convergence study was performed to determine $N=50000$ sufficient to converge all eigenvalues to $<10^{-12}$ a.u., see Figs. $3 \mathrm{a}$ and $3 \mathrm{~b}$. 


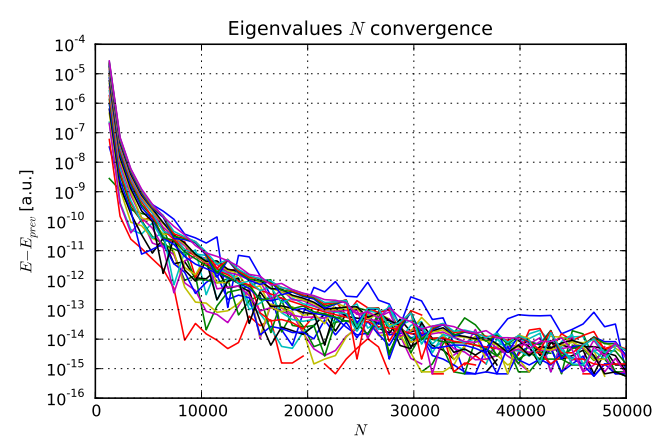

(a) $N$ study eigenvalues

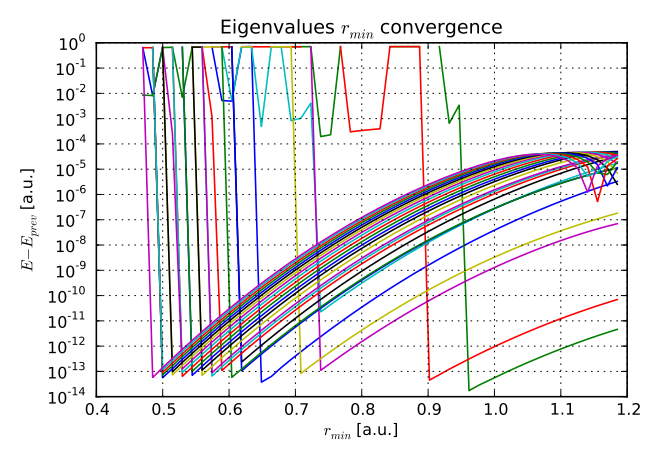

(c) $r_{\min }$ study eigenvalues

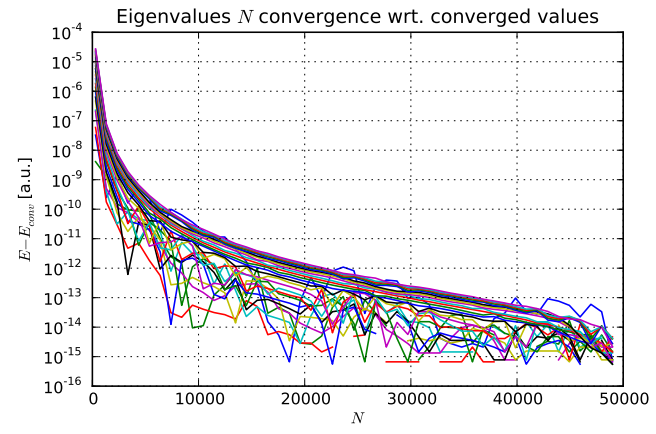

(b) $N$ study eigenvalues

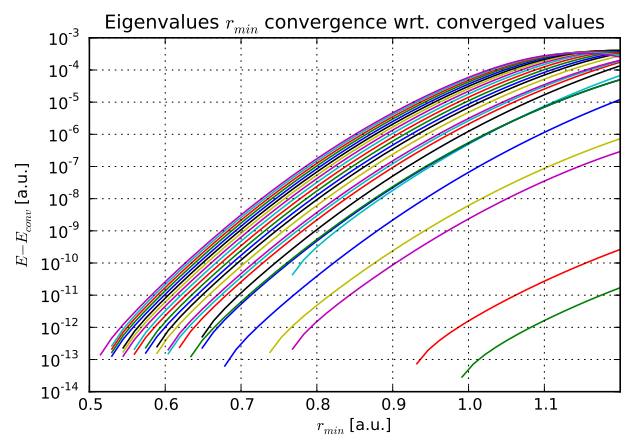

(d) $r_{\min }$ study eigenvalues

Fig. 3. Double minimum potential convergence studies.

Convergence with respect to $r_{\min }$ was then studied with $N=50000$, see Fig. 3c, from which a converged energy and $r_{\min }$ were determined for each eigenvalue, as shown in Table 7 . Fig. 3d shows the convergence with respect to these converged energies. From these, we find eigenvalues stable to $<10^{-8}$ a.u. with respect to both $r_{\min }$ and $N$.

To verify the results, we compared to independent high-order finite-element based calculations [44] on the interval [0,12] a.u., which require no assumptions of asymptotic forms or determination of $r_{\min }$. Agreement was obtained for all eigenvalues to $<10^{-8}$ a.u. $\left(<10^{-2} \mathrm{~cm}^{-1}\right)$. To compare to [20], we implemented the finite difference method described there and used a uniform mesh with $N=100000$ points on the interval $[0,12]$ a.u. The results are shown in the Table 7. As can be seen, agreement is again obtained to $<10^{-8}$ a.u. $(<$ $10^{-2} \mathrm{~cm}^{-1}$ ). We thus obtain agreement of all eigenvalues to $<10^{-8}$ a.u. by three independent methods and codes. Remaining differences from [45] and [20] are thus most likely attributable to differences in interpolation, choice of $r_{\text {min }}$, and/or convergence with respect to other computational parameters. 


\begin{tabular}{|c|c|c|c|c|c|}
\hline$v$ & $r_{\min }[\mathrm{a} . \mathrm{u}]$. & $E$ [a.u.] (dftatom) & $E$ [a.u.] (FD) & $E\left[\mathrm{~cm}^{-1}\right]$ (dftatom) & $E\left[\mathrm{~cm}^{-1}\right](\mathrm{FD})$ \\
\hline 0 & 0.679 & -0.71248345 & -0.71248345 & 19200.40 & 19200.40 \\
\hline 1 & 0.991 & -0.71169411 & -0.71169411 & 19027.16 & 19027.16 \\
\hline 2 & 0.932 & -0.70624880 & -0.70624880 & 17832.05 & 17832.05 \\
\hline 3 & 0.768 & -0.70187763 & -0.70187763 & 16872.69 & 16872.69 \\
\hline 4 & 0.768 & -0.70106161 & -0.70106161 & 16693.59 & 16693.59 \\
\hline 5 & 0.738 & -0.69617697 & -0.69617697 & 15621.54 & 15621.54 \\
\hline 6 & 0.649 & -0.69265160 & -0.69265160 & 14847.81 & 14847.81 \\
\hline 7 & 0.649 & -0.69127773 & -0.69127773 & 14546.28 & 14546.28 \\
\hline 8 & 0.634 & -0.68736218 & -0.68736218 & 13686.92 & 13686.92 \\
\hline 9 & 0.619 & -0.68429008 & -0.68429008 & 13012.67 & 13012.67 \\
\hline 10 & 0.604 & -0.68145547 & -0.68145547 & 12390.54 & 12390.54 \\
\hline 11 & 0.604 & -0.67811006 & -0.67811006 & 11656.31 & 11656.31 \\
\hline 12 & 0.589 & -0.67481518 & -0.67481519 & 10933.17 & 10933.17 \\
\hline 13 & 0.589 & -0.67163329 & -0.67163330 & 10234.82 & 10234.82 \\
\hline 14 & 0.574 & -0.66844493 & -0.66844493 & 9535.06 & 9535.06 \\
\hline 15 & 0.574 & -0.66526724 & -0.66526725 & 8837.64 & 8837.64 \\
\hline 16 & 0.559 & -0.66215075 & -0.66215075 & 8153.65 & 8153.65 \\
\hline 17 & 0.559 & -0.65910546 & -0.65910546 & 7485.28 & 7485.28 \\
\hline 18 & 0.544 & -0.65612571 & -0.65612571 & 6831.30 & 6831.30 \\
\hline 19 & 0.544 & -0.65321351 & -0.65321351 & 6192.15 & 6192.15 \\
\hline 20 & 0.544 & -0.65037671 & -0.65037671 & 5569.54 & 5569.54 \\
\hline 21 & 0.530 & -0.64762174 & -0.64762175 & 4964.90 & 4964.90 \\
\hline 22 & 0.530 & -0.64495237 & -0.64495238 & 4379.04 & 4379.04 \\
\hline 23 & 0.530 & -0.64237186 & -0.64237186 & 3812.68 & 3812.68 \\
\hline 24 & 0.530 & -0.63988486 & -0.63988486 & 3266.85 & 3266.85 \\
\hline 25 & 0.515 & -0.63749749 & -0.63749749 & 2742.88 & 2742.88 \\
\hline
\end{tabular}

Table 7

Computed Schrödinger eigenvalues for double minimum potential. The $r_{\min }$ column shows values that were used for the shooting method. The finite difference (FD) method was used on the interval $[0,12]$.

\subsection{Uranium}

We now consider full, self-consistent nonrelativistic (LDA) and relativistic (RLDA) Kohn-Sham calculations of the ground state of uranium: a stringent test case, as numerous eigenstates are required, with tightly bound, highly oscillatory $s$ states, spanning energies from $\sim-0.1$ to $\sim-4,000$ a.u.

We use the same electronic configuration as in $[27,28]$ : for the nonrelativistic calculation, $1 s^{2}, 2 s^{2}, 2 p^{6}, 3 s^{2}, 3 p^{6}, 3 d^{10}, 4 s^{2}, 4 p^{6}, 4 d^{10}, 4 f^{14}, 5 s^{2}, 5 p^{6}, 5 d^{10}$, $5 f^{3}, 6 s^{2}, 6 p^{6}, 6 d^{1}, 7 s^{2}$; and for the relativistic calculation, the occupation of each $l$-shell is split according to the degeneracy of $j=l+\frac{1}{2}$ and $j=l-\frac{1}{2}$ subshells.

We first determine sufficiently large $r_{\max }$ that the associated error (due to confining wavefunctions and potentials) is below the level of other sources in the calculation. We start by setting $r_{\min }, r_{\max }, a$, and $N$ to typical values consistent with previous findings [27]. We then increase $N$ to converge to the limit of finite precision. Then, increasing $r_{\max }$ in LDA (Figs. 4b and 4c) and RLDA (Figs. 5b and 5c) calculations (with perturbation correction turned off), considering both eigenvalues and total energy, shows that $r_{\max }=50$ a.u. 
is a well converged value for both LDA and RLDA, consistent with previous findings [27].

The aim of the next $N$ study is then to find $N$ (and $a$ ) such that the total energy is converged for broad range of $r_{\min }$, so that such $N$ can be used for the $r_{\text {min }}$ study. In theory, by simply increasing $N$, the total energy must eventually converge, but for small $a$ (low concentration of grid points around the origin), the required $N$ might be very high. There is some coupling of $r_{\min }$ and $a$ due to closer approach to the singularity at $r=0$ for smaller $r_{\min }$ : smaller $r_{\text {min }}$ requires somewhat larger $a$ for a given $N$ and accuracy. It was found that using $a=10^{9}$, full convergence is achieved for all $r_{\min }>10^{-14}$ with $N=50000$.

The initial point $r_{\min }>0$ determines the accuracy of the asymptotics used to start the radial integrations, which are exact only in the limit $r \rightarrow 0$. The goal of the $r_{\min }$ study is to find fully converged $r_{\min }$ such that the associated error is at the limit of finite precision; so that decreasing further does not improve results. To do so, the fully converged $a$ and $N$ are taken from the initial $N$ study, then converged total energies are calculated for all $r_{\min }$ from $10^{-14}$ to $10^{-5}$.

Fig. 4a shows the $r_{\text {min }}$ study for the LDA calculation. As can be seen, the total energy is converged for $r_{\min }=10^{-7}$ and decreasing $r_{\min }$ further, the error indicators remain below the $5 \cdot 10^{-9}$ level. Choosing $r_{\min }$ smaller than $10^{-7}$ does not increase accuracy further. In general, choosing $r_{\min }$ as large as possible avoids the need for excessive grid points to resolve rapidly varying potentials, densities, and wavefunctions in the vicinity of the Coulomb singularity at $r=0$. As such, $r_{\min }=10^{-7}$ is used, as it gives sufficiently accurate asymptotics such that the associated errors are reduced to the limits of finite precision (which eliminates $r_{\min }$ from consideration when choosing other parameters), is consistent with values adopted previously [27], and is not so small as to require excessive grid points around the origin.

Fig. 5a shows the $r_{\text {min }}$ study for the RLDA calculation. Using the same procedure as for the LDA calculation, $r_{\min }=10^{-8}$ was chosen.

Now the optimal mesh for $10^{-6}$ a.u. accuracy in total energy is found by setting $r_{\min }$ and $r_{\max }$ to converged values and varying the mesh gradation $a$ to minimize the number of elements $N$ required to achieve the specified accuracy. Proceeding in this way, we find optimal mesh parameters as in Table 2 for $10^{-6}$ a.u. accuracy in the total energy of uranium.

Having determined converged $r_{\min }, r_{\max }$, and optimized $a$ (Table 2), we take $N$ to convergence (Fig. $4 \mathrm{~d}$ ) to find converged total energy

$$
E_{t o t}=-25658.41788885 \text { a.u. }
$$




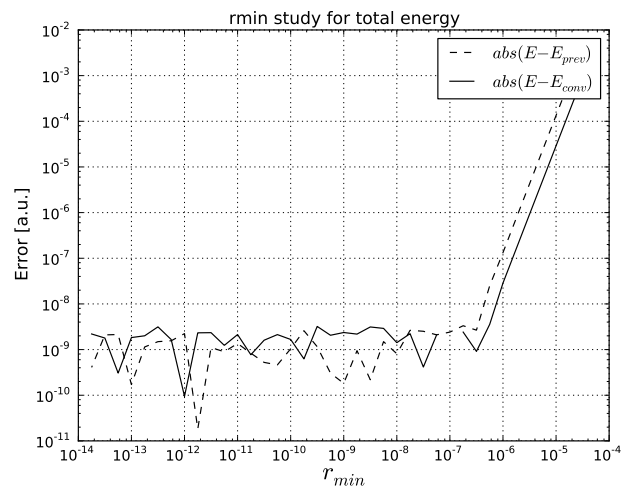

(a) $r_{\min }$ study total energy

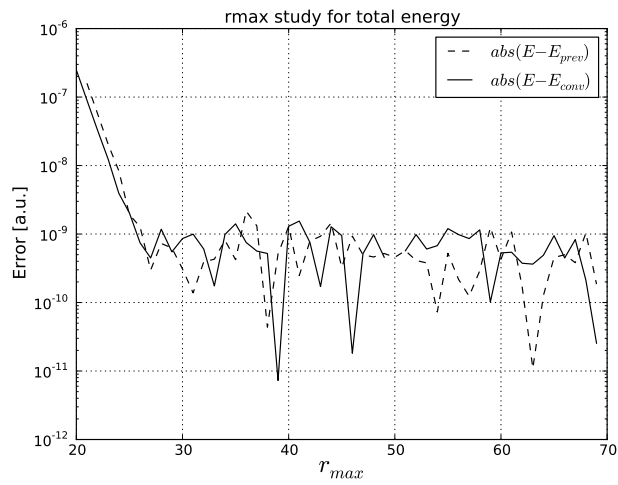

(c) $r_{\max }$ study total energy

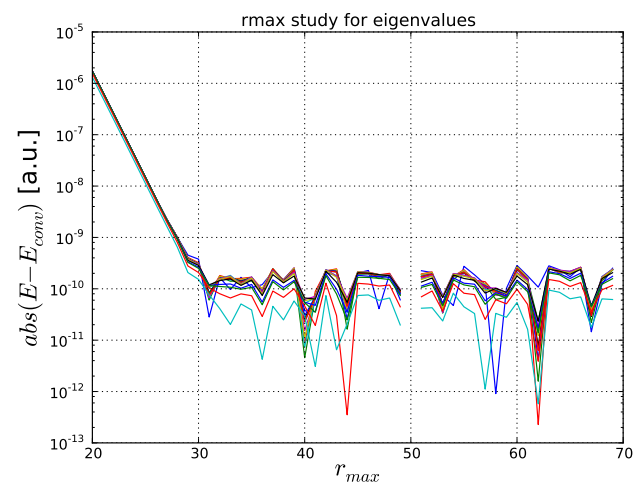

(b) $r_{\max }$ study eigenvalues

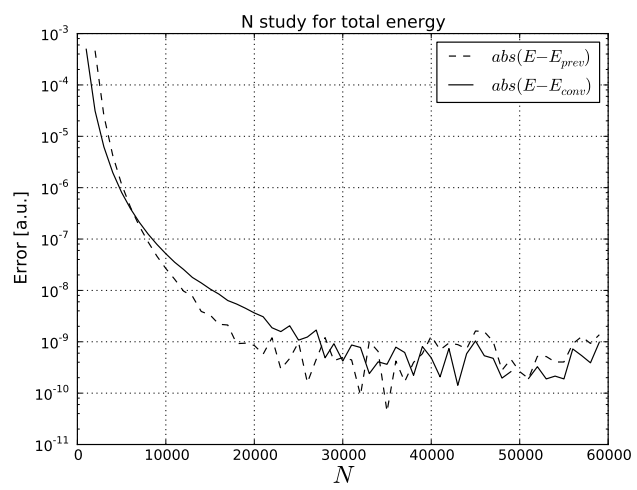

(d) $N$ study total energy

Fig. 4. Non-relativistic (LDA) density functional theory (DFT) convergence studies for uranium.

and orbital energies (Table 8) with error $<10^{-8}$ a.u. for the LDA calculation.

Proceeding similarly for the RLDA calculation, we take $N$ to convergence (Fig. 5d) to find converged total energy

$$
E_{t o t}=-28001.13232548 \text { a.u. }
$$

and orbital energies (Table 9) with error $<10^{-8}$ a.u. for the RLDA calculation also.

\subsubsection{Optimal $N$}

In the previous section, for converged $r_{\min }, r_{\max }$, and optimized $a$ (Table 2 ), sufficient $N$ was determined to achieve $10^{-6}$ accuracy in total energies for uranium. In this section, we determine sufficient $N$ for a series of accuracies, from $10^{-3}$ to $10^{-8}$ a.u., for all elements $Z=1-92$.

Fig. 6a shows the required $N$ for LDA (Schrödinger) and Fig. $6 \mathrm{~b}$ for RLDA 


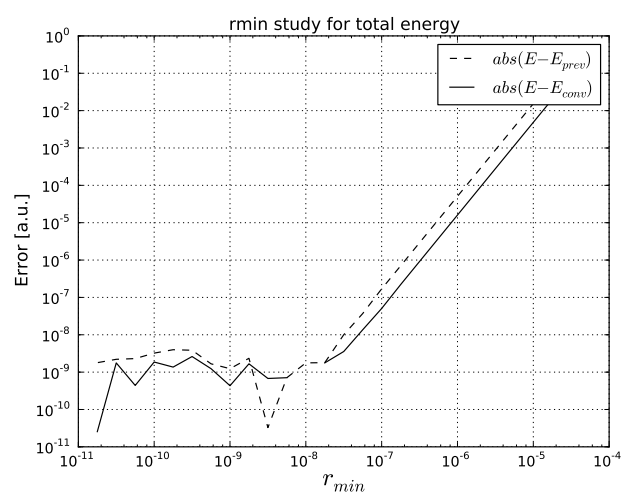

(a) $r_{\min }$ study total energy

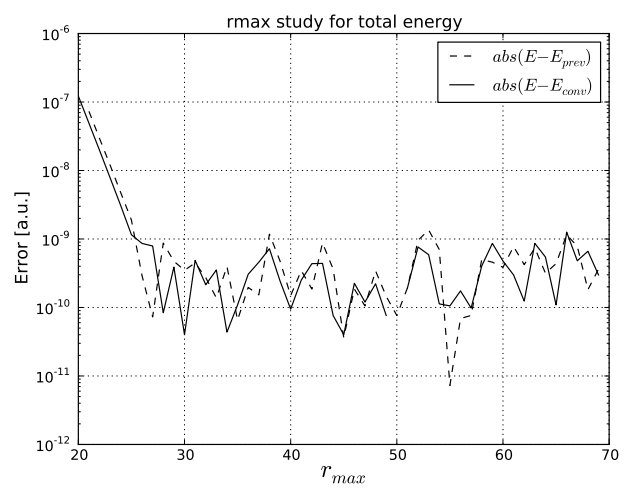

(c) $r_{\max }$ study total energy

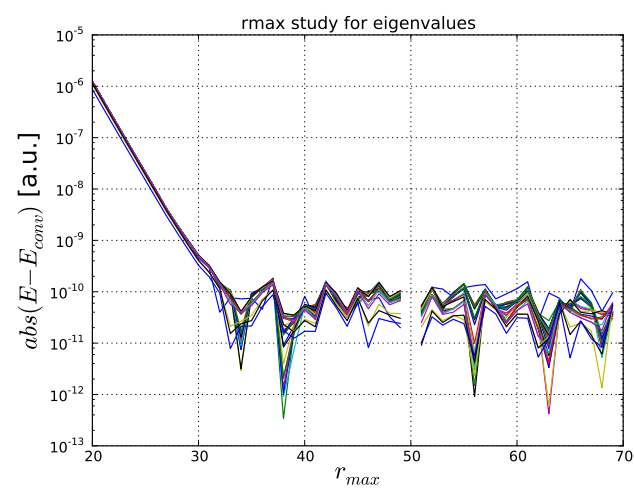

(b) $r_{\max }$ study eigenvalues

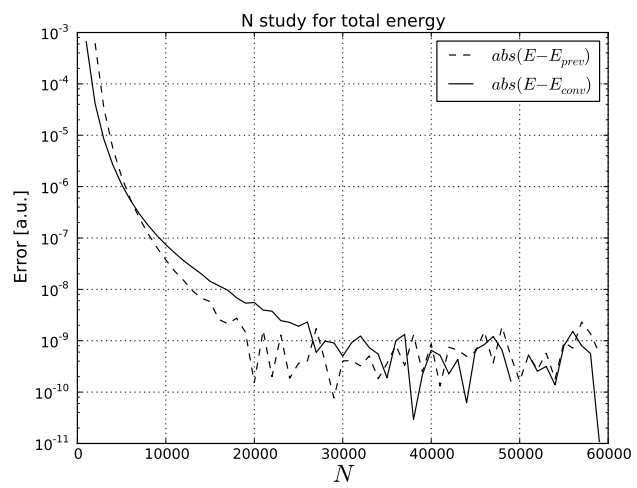

(d) $N$ study total energy

Fig. 5. Relativistic (RLDA) density functional theory (DFT) convergence studies for uranium.

Table 8

\begin{tabular}{cr} 
state & eigenvalue [a.u.] \\
\hline $1 \mathrm{~s}$ & -3689.35513984 \\
$2 \mathrm{~s}$ & -639.77872809 \\
$2 \mathrm{p}$ & -619.10855018 \\
$3 \mathrm{~s}$ & -161.11807321 \\
$3 \mathrm{p}$ & -150.97898016 \\
$3 \mathrm{~d}$ & -131.97735828 \\
$4 \mathrm{~s}$ & -40.52808425 \\
$4 \mathrm{p}$ & -35.85332083 \\
$4 \mathrm{~d}$ & -27.12321230 \\
$4 \mathrm{f}$ & -15.02746007 \\
$5 \mathrm{~s}$ & -8.82408940 \\
$5 \mathrm{p}$ & -7.01809220 \\
$5 \mathrm{~d}$ & -3.86617513 \\
$5 \mathrm{f}$ & -0.36654335 \\
$6 \mathrm{~s}$ & -1.32597632 \\
$6 \mathrm{p}$ & -0.82253797 \\
$6 \mathrm{~d}$ & -0.14319018 \\
$7 \mathrm{~s}$ & -0.13094786
\end{tabular}

Computed non-relativistic (LDA) eigenvalues for uranium $\left(10^{-8}\right.$ a.u. accurate). 
Table 9

\begin{tabular}{lr} 
state & eigenvalue [a.u.] \\
\hline $1 s_{1 / 2}$ & -4223.41902045 \\
$2 s_{1 / 2}$ & -789.48978233 \\
$2 p_{3 / 2}$ & -761.37447597 \\
$2 p_{1 / 2}$ & -622.84809456 \\
$3 s_{1 / 2}$ & -199.42980564 \\
$3 p_{3 / 2}$ & -186.66371312 \\
$3 p_{1 / 2}$ & -154.70102667 \\
$3 d_{5 / 2}$ & -134.54118029 \\
$3 d_{3 / 2}$ & -128.01665738 \\
$4 s_{1 / 2}$ & -50.78894806 \\
$4 p_{3 / 2}$ & -45.03717129 \\
$4 p_{1 / 2}$ & -36.68861049 \\
$4 d_{5 / 2}$ & -27.52930624 \\
$4 d_{3 / 2}$ & -25.98542891 \\
$4 f_{7 / 2}$ & -13.88951423 \\
$4 f_{5 / 2}$ & -13.48546969 \\
$5 s_{1 / 2}$ & -11.29558710 \\
$5 p_{3 / 2}$ & -9.05796425 \\
$5 p_{1 / 2}$ & -7.06929564 \\
$5 d_{5 / 2}$ & -3.79741623 \\
$5 d_{3 / 2}$ & -3.50121718 \\
$5 f_{7 / 2}$ & -0.14678839 \\
$5 f_{5 / 2}$ & -0.11604717 \\
$6 s_{1 / 2}$ & -1.74803995 \\
$6 p_{3 / 2}$ & -1.10111900 \\
$6 p_{1 / 2}$ & -0.77578418 \\
$6 d_{5 / 2}$ & -0.10304082 \\
$6 d_{3 / 2}$ & -0.08480202 \\
$7 s_{1 / 2}$ & -0.16094728
\end{tabular}

Computed relativistic (RLDA) eigenvalues for uranium $\left(10^{-8}\right.$ a.u. accurate).

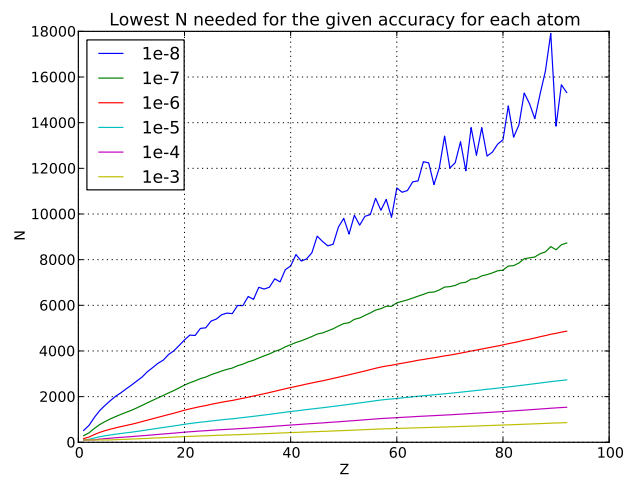

(a) Schrödinger (LDA)

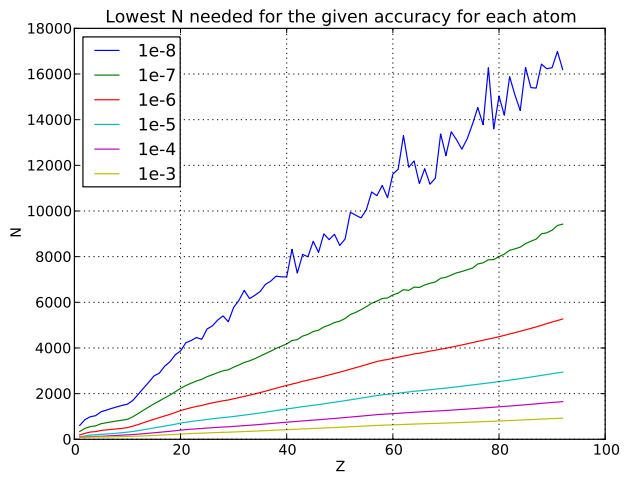

(b) Dirac (RLDA)

Fig. 6. Accuracy graphs for non-relativistic (LDA) and relativistic (RLDA) density functional theory (DFT) calculations.

\section{(Dirac) Kohn-Sham equations.}

For simplicity, we use the same converged $r_{\min }, r_{\max }$, and optimized $a$ (Table 2) for all atoms and just vary $N$ for each atom to find a sufficient value for the specified accuracy. 


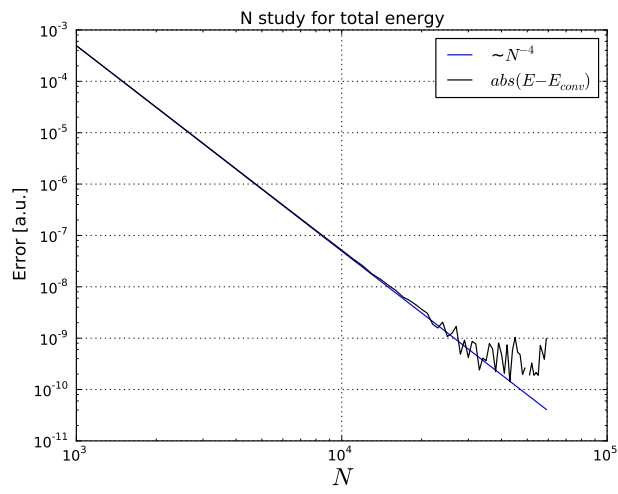

(a) Schrödinger

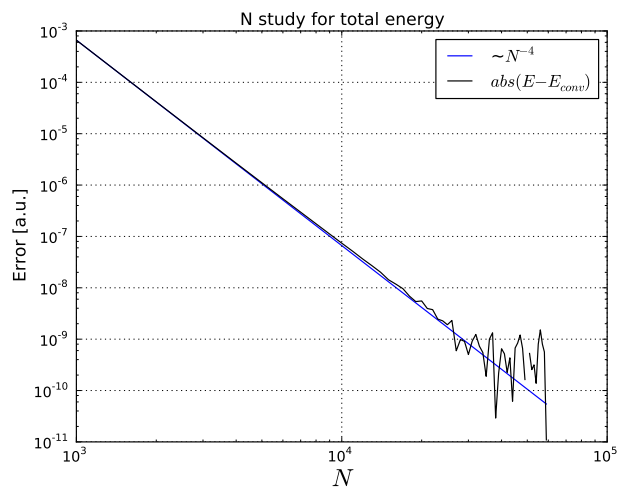

(b) Dirac

Fig. 7. Non-relativistic (LDA) and relativistic (RLDA) convergence rates from the $N$-studies for total energy plotted on a log-log scale.

The $N$ shown in Figs. $6 \mathrm{a}$ and $6 \mathrm{~b}$ is mainly for use as a starting point. Sufficient $N$ will vary somewhat with the choice of exchange-correlation, and very much so with the choice of $r_{\min }, r_{\max }$, and $a$. If any of these are changed, as may be desired to find more optimal values for different atoms and/or accuracy requirements, a new $N$ study will be required to determine sufficient $N$. If, however, the converged $r_{\min }, r_{\max }$, and $a$ from Table 2 are used, then convergence can be attained for any atom to the limits of machine precision by simply increasing $N$.

By setting $N=50000$ with converged $r_{\min }, r_{\max }$, and $a$ from Table 2 , fully converged total energies and Kohn-Sham eigenvalues are obtained, and used to compute errors for Figs. 6a and $6 \mathrm{~b}$.

\subsubsection{Numerical error}

The intrinsic numerical error of the Schrödinger, Dirac, and Poisson solvers is $O\left(h^{4}\right)$, where $h$ is the mesh spacing, since we use 4th-order Runge-Kutta and Adams methods. For the uranium test case, the convergence rate in terms of the number of mesh points $N(\propto 1 / h)$ can be estimated from the $N$ convergence studies (Fig. 4d and 5d) plotted on a log-log scale, as shown in Figs. $7 \mathrm{a}$ and $7 \mathrm{~b}$. For comparison, the line $N^{-4}$ is plotted also (shifted to agree at lowest $N)$. As can be seen, the actual convergence follows $N^{-4}$ almost exactly, indicating an error of $O\left(h^{4}\right)$, consistent with the order of methods employed. 


\subsubsection{Comparison to current benchmarks}

Using the meshes from Table 2 with converged $N=50000$, the computed Kohn-Sham total energies and eigenvalues were compared with current benchmark values $[27,28]$ for all atoms $Z=1-92$ for LDA (Schrödinger) and RLDA (Dirac) Kohn-Sham calculations. It was found that the computed total energies were all within $5.29 \cdot 10^{-7}$ of benchmark values for LDA and within $5.31 \cdot 10^{-7}$ for RLDA; and that the computed eigenvalues were all within $1.24 \times 10^{-6}$ of benchmark values for LDA and within $1.37 \times 10^{-6}$ for RLDA.

Reference [27] claims $10^{-6}$ a.u. absolute accuracy for Kohn-Sham total energies and finds maximum deviations among different codes of $2 \cdot 10^{-6}$ for associated eigenvalues. Our results, converged to $<10^{-8}$ a.u., verify that the total energies reported in [27] indeed have absolute accuracy $10^{-6}$ a.u. or better; and furthermore, that the eigenvalues in [27] in fact have absolute accuracy $2 \cdot 10^{-6}$ a.u. or better.

\section{$5 \quad$ Summary and conclusions}

We have presented a robust and general solver for the solution of the radial Schrödinger, Dirac, and Kohn-Sham equations of density functional theory; and provided a modular, portable, and efficient Fortran 95 implementation, dftatom, along with interfaces to other languages and full suite of examples and tests. The solver brings together ideas from many different codes developed over the decades and in addition employs ideas such as outward Poisson integration for increased accuracy in the core region and perturbation with fallback to bisection for speed and robustness. The solver can accommodate any potential, whether singular Coulombic or finite, and any mesh, whether linear, exponential, or otherwise. We have demonstrated the flexibility and accuracy of the associated code with solutions of Schrödinger and Dirac equations for Coulombic, harmonic oscillator, and double minimum potentials; and solutions of Kohn-Sham and Dirac-Kohn-Sham equations for the challenging case of uranium, obtaining energies accurate to $10^{-8}$ a.u., thus verifying and refining by two orders of magnitude current benchmarks [27]. We have shown detailed convergence studies in each case, providing mesh parameters to facilitate straightforward convergence to any desired accuracy by simply increasing the number of mesh points.

At all points in the design of the associated code, we have tried to emphasize simplicity and modularity so that the routines provided can be straightforwardly employed for a range of applications purposes, while retaining high efficiency. We have made the code available as open source to facilitate distribution, modification, and use as needed. We expect the present solvers will 
be of benefit to a range of larger-scale electronic structure methods which rely on atomic structure calculations and/or radial integrations more generally as key components.

\section{Acknowledgements}

We would like to thank Don Hamann and Mark Stiles for discussing and sharing their codes with us, as well as Eric Shirley for pointing us to other codes. We thank Charlotte Froese Fischer and Zbigniew Romanowski for helpful discussions and Peter Winkler for a critical reading of the manuscript. Finally, we thank the referees for a number of valuable suggestions which improved the manuscript considerably.

This work performed, in part, under the auspices of the U.S. Department of Energy by Lawrence Livermore National Laboratory under Contract DEAC52-07NA27344. This research was partly supported by the LC06040 research center project and GACR 101/09/1630 of the Czech Science Foundation.

\section{References}

[1] Martin, R. M., Electronic Structure: Basic Theory and Practical Methods, Cambridge University Press, 2005.

[2] Wilson, B., Sonnad, V., Sterne, P., and Isaacs, W., J. Quant. Spectrosc. Radiat. Transf. 99 (2006) 658.

[3] Butler, W. H., Dederichs, P. H., Gonis, A., and Weaver, R. L., Application of Multiple Scattering Theory to Materials Science, Materials Research Society, Pittsburg, Penn., 1992.

[4] Skriver, H. L., The LMTO Method, Springer, Berlin, 1984.

[5] Singh, D. J. and Nordstrom, L., Planewaves, Pseudopotentials, and the LAPW Method, Springer, New York, 2nd edition, 2006.

[6] Artacho, E. et al., J. Phys.: Cond. Matter 20 (2008), 2nd Workshop on Theory Meets Industry, Erwin Schrodinger Inst, Vienna, Austria, Jun 12-14, 2007.

[7] Blöchl, P. E., Phys. Rev. B 50 (1994) 17953.

[8] Marsman, M. and Kresse, G., J. Chem. Phys. 125 (2006).

[9] Vackář, J. and Šimůnek, A., Phys. Rev. B 67 (2003). 
[10] Desclaux, J. P., Mayers, D. F., and O’Brien, F., J. Phys. B: At. Mol. Phys. 4 (1971) 631.

[11] Hamann, D. R., Phys. Rev. B 40 (1989) 2980.

[12] Jonsson, P., He, X., Froese Fischer, C., and Grant, I. P., Comput. Phys. Commun. 177 (2007) 597.

[13] Froese Fischer, C., Comput. Phys. Commun. 64 (1991) 431.

[14] Tackett, A. R., Holzwarth, N. A. W., and Matthews, G. E., Comput. Phys. Commun. 135 (2001) 348.

[15] Jia, W. et al., Comput. Phys. Commun. 184 (2013) 9.

[16] Dewhurst, K., Elk, http://elk.sourceforge.net/, 2012.

[17] Pask, J. E. and Sterne, P. A., Model. Simul. Mater. Sci. Eng. 13 (2005) R71.

[18] Press, W. H., Teukolsky, S. A., Vetterling, W. T., and Flannery, B. P., Numerical Recipes: The Art of Scientific Computing, Cambridge University Press, New York, 3 edition, 2007.

[19] Cayford, J., Fimple, W., Unger, D., and White, S., J. Comput. Phys. 16 (1974) 259.

[20] Tobin, F. L. and Hinze, J., J. Chem. Phys. 63 (1975) 1034.

[21] Biegler-König, F. and Hinze, J., J. Comput. Phys. 67 (1986) 290.

[22] Andrae, D. and Hinze, J., Int. J. Quantum Chem. 63 (1997) 65.

[23] Andrae, D., Reiher, M., and Hinze, J., Int. J. Quantum Chem. 76 (2000) 473.

[24] Andrae, D., Brodbeck, R., and Hinze, J., Int. J. Quantum Chem. 82 (2001) 227.

[25] Fischer, C. F. and Zatsarinny, O., Comput. Phys. Commun. 180 (2009) 879.

[26] Grant, I. P., J. Phys. B: At. Mol. Opt. Phys. 42 (2009).

[27] Kotochigova, S., Levine, Z. H., Shirley, E. L., Stiles, M. D., and Clark, C. W., Phys. Rev. A 55 (1997) 191.

[28] NIST, Atomic reference data for electornic structure calculations, http:// physics.nist.gov/PhysRefData/DFTdata/, 1997.

[29] Čertík, O., Pask, J. E., and Vackář, J., dftatom, http://sourceforge.net/ projects/dftatom/, 2012.

[30] Johnson, W. R., Lectures on atomic physics, http://nd.edu/ johnson/ Publications/book.pdf, 2006.

[31] Strange, P., Relativistic Quantum Mechanics, Cambridge University Press, 1998. 
[32] Zabloudil, J., Hammerling, R., Szunyogh, L., and Weinberger, P., Electron Scattering in Solid Matter, Springer-Verlag Berlin, 2005.

[33] Grant, I. P., J. Phys. B: At. Mol. Opt. Phys. 41 (2008) 1.

[34] Oulne, M., Int. Rev. Phys. 4 (2010) 349.

[35] Vosko, S. H., Wilk, L., and Nusair, M., Can. J. Phys. 58 (1980).

[36] MacDonald, A. H. and Vosko, S. H., J. Phys. C: Solid St. Phys. 12 (1979).

[37] Ridley, E. C., Proc. Camb. Phil. Soc. 51 (1955) 702.

[38] Douglas, A. S., Proc. Camb. Phil. Soc. 52 (1955) 636.

[39] Hartree, D. R., The calculation of atomic structures., John Wiley \& Sons, Inc., New York, 1957.

[40] Mayers, D. F., Proc. R. Soc. Lond. A 241 (1957) 93.

[41] Torrance, C. C., Phys. Rev. 46 (1934) 388.

[42] Yost, W. J., Phys. Rev. 58 (1940) 557.

[43] Cohen, E. R. and Taylor, B. N., Rev. Mod. Phys. 59 (1987) 1121.

[44] Čertík, O. and Pask, J. E., High-order finite element method for atomic structure calculations, In preparation.

[45] Kolos, W. and Wolniewicz, L., J. Chem. Phys. 50 (1969) 3228.

[46] Lin, C. S., J. Chem. Phys. 60 (1974) 4660.

[47] Connerade, J. P. and Dolmatov, V. K., J. Phys. B: At. Mol. Opt. Phys. 31 (1998) 3557. 